\title{
THE PARTIAL POSITIVITY OF THE CURVATURE IN RIEMANNIAN SYMMETRIC SPACES
}

\author{
Xusheng Liu \\ School of Mathematical Sciences, \\ Fudan University, Shanghai 200433, China \\ xshliu@fudan.edu.cn
}

\begin{abstract}
In this paper, we determine the partial positivity(resp., negativity) of the curvature of all irreducible Riemannian symmetric spaces. From the classifications of abstract root systems and maximal subsystems, we can give the calculations for symmetric spaces both in classical types and in exceptional types.
\end{abstract}

\section{INTRODUCTION}

We recall the definition of $s$-positive curvature from $\mathrm{Wu}$. A Riemannian manifold $M$ has $s$-positive(resp., s-negative) curvature if and only if for each $x \in M$ and for any $(s+1)$ orthonormal vectors $\left\{e_{0}, e_{1}, \cdots, e_{s}\right\}$ in $M_{x}, \sum_{i=1}^{s} K\left(e_{0}, e_{i}\right)>0$ (resp., $<0)$, where $K\left(e_{0}, e_{i}\right)$ denotes the sectional curvature of the plane spanned by $e_{0}$ and $e_{i}$. The 1-positive curvature is equivalent to positive sectional curvature, and (n-1)-positive curvature is equivalent to positive Ricci curvature.

The manifolds which have $s$-positive(or negative) curvature have some topological implications as well as their geometric properties. These manifolds were studied

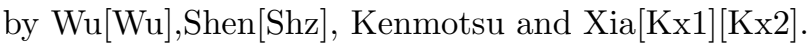

Among them it was shown in $\underline{S h z}$ that if a proper open manifold $M$ has $s$ positive curvature then $M$ has the homotopy type of a $C W$ complex with cells each of dimension $\leq s-1$. In particular, $H_{i}(M, \mathbb{Z})=0$, for $i \geq s$.

Frankel [Fr1] showed that two compact totally geodesic submanifolds in an ndimensional complete Riemannian manifold $\mathrm{N}$ of positive sectional curvature must intersect if the sum of their dimension is greater than or equal to $\mathrm{n}$; and he proved Fr2 that if $\mathrm{V}$ is an $\mathrm{r}$-dimensional compact totally geodesic immersed submanifold of $\mathrm{N}$ with $2 r>n$, then the homomorphism of fundamental group $\pi_{1}(V) \rightarrow \pi_{1}(N)$ is surjective.

These results had been generalized in the case of manifolds with partially positive curvature by Kenmotsu and Xia in $\mathrm{Kx2}$. They showed in an n-dimensional complete connected Riemannian manifold with k-nonnegative curvature, let $\mathrm{V}$ and $\mathrm{W}$ be two complete immersed totally geodesic submanifolds of dimensions $\mathrm{r}$ and $\mathrm{s}$, let one of $\mathrm{V}$ and $\mathrm{W}$ be compact and suppose $\mathrm{N}$ has k-positive curvature either at all points of $\mathrm{V}$ or at all points of $\mathrm{W}$, if $r+s \geq n+k-1$ then $\mathrm{V}$ and $\mathrm{W}$ must intersect. As the same time they also proved that if $\mathrm{V}$ is a $\mathrm{r}$-dimensional totally geodesic submanifold with $2 r \geq n+k-1$, then the homomorphism of fundamental group $\pi_{1}(V) \rightarrow \pi_{1}(N)$ is surjective. These show that there exist topological obstructions for the existence of higher dimensional totally geodesic submanifolds in the Riemannian manifolds with partially positive curvature. 
Those results show that the notion of s-positivity(or negativity) is more subtle curvature condition. Naturally, determining all the s-values for irreducible Riemannian symmetric spaces of compact type is an interesting problem. This has been started by Lee Lee2. She gave the s-values for the symmetric spaces of classical types by using of the matrix representation.

What about the exceptional cases? Concerning more and more physicists pay attention to the exceptional geometry, we calculate the partial positivity of the curvature of all irreducible Riemannian symmetric spaces of compact type. If $M$ has s-positive curvature, the dual of $M$ is a simply connected irreducible symmetric space of noncompact type which has s-negative curvature.

Our method is different from hers and we can deal with both classical types as well as the exceptional types. For the classical types we recover Lee's result in [Lee2]. Our main result is the following table.

Table 1.1

\begin{tabular}{|c|c|c|c|c|}
\hline Type & compact type & rank & dimension & $\mathrm{s}$ \\
\hline$A I$ & $S U(n) / S O(n)$ & $n-1$ & $\frac{1}{2}(n-1)(n+2)$ & $\frac{n(n-1)}{2}$ \\
\hline$A I I$ & $S U(2 n) / S p(n)$ & $n-1$ & $(n-1)(2 n+1)$ & $(n-1)(2 n-3)$ \\
\hline$A I I I$ & $\left.S U(p+q)) / S\left(U_{p} \times U_{q}\right)\right)$ & $\min (p, q)$ & $2 p q$ & $1+2(p-1)(q-1)$ \\
\hline$B D I$ & $S O(p+q)) / S O(p) \times S O(q)$ & $\min (p, q)$ & $p q$ & $1+(p-1)(q-1)$ \\
\hline$D I I I$ & $S O(2 n)) / U(n)$ & {$\left[\frac{1}{2} n\right]$} & $n(n-1)$ & $1+(n-2)(n-3)$ \\
\hline$C I$ & $S p(n) / U(n)$ & $n$ & $n(n+1)$ & $1+n(n-1)$ \\
\hline$C I I$ & $S p(p+q)) / S p(p) \times S p(q)$ & $\min (p, q)$ & $4 p q$ & $1+4(p-1)(q-1)$ \\
\hline$E I$ & $\left(\mathfrak{e}_{6(-78)}, \mathfrak{s p}(4)\right)$ & 6 & 42 & 26 \\
\hline$E I I$ & $\left(\mathfrak{e}_{6(-78)}, \mathfrak{s u}(6)+\mathfrak{s u}(2)\right)$ & 4 & 40 & 19 \\
\hline$E I I I$ & $\left(\mathfrak{e}_{6(-78)}, \mathfrak{s o}(10)+\mathbb{R}\right)$ & 2 & 32 & 11 \\
\hline$E I V$ & $\left(\mathfrak{e}_{6(-78)}, \mathfrak{f}(4)\right)$ & 2 & 26 & 10 \\
\hline$E V$ & $\left(\mathfrak{e}_{7(-133)}, \mathfrak{s u}(8)\right)$ & 7 & 70 & 43 \\
\hline$E V I$ & $\left(\mathfrak{e}_{7(-133)}, \mathfrak{s o}(12)+\mathfrak{s u}(2)\right)$ & 4 & 64 & 31 \\
\hline$E V I I$ & $\left(\mathfrak{e}_{7(-133)}, \mathfrak{e}_{6}+\mathbb{R}\right)$ & 3 & 54 & 27 \\
\hline$E V I I I$ & $\left(\mathfrak{e}_{8(-248)}, \mathfrak{s o}(16)\right)$ & 8 & 128 & 71 \\
\hline$E I X$ & $\left(\mathfrak{e}_{8(-248)}, \mathfrak{e}_{7}+\mathfrak{s u}(2)\right)$ & 4 & 112 & 55 \\
\hline$F I$ & $\left(\mathfrak{f}_{4(-52)}, \mathfrak{s p}(3)+\mathfrak{s u}(2)\right)$ & 4 & 28 & 13 \\
\hline$F I I$ & $\left(\mathfrak{f}_{4(-52)}, \mathfrak{s o}(9)\right)$ & 1 & 16 & 1 \\
\hline$G$ & $\left(\mathfrak{g}_{2(-14)}, \mathfrak{s u}(2)+\mathfrak{s u}(2)\right)$ & 2 & 8 & 3 \\
\hline
\end{tabular}

Remark 1.1. We note some exceptional cases.

(i) if $r=\operatorname{rank}(M)=1$, then $s=r=1$.

(ii)For $A I I I, p=q=2, s=4$.

(iii)For BDI, $p=q=2, s=3 ; p=q=3, s=6$.

Acknowledgements. I am grateful to my supervisor, Prof. Y.L.Xin, for his constant guidance. He brought [Lee2] to my notice, and suggested me the method of Lie algebra. He also pointed out some mistakes in the course of this work. 


\section{Abstract ROOT SYSTEM AND SUBSystem}

An abstract root system in a finite dimensional real inner product space $V$ with inner product $\langle$,$\rangle is a finite set \Delta$ (whose element is called a root) of $V-\{0\}$ such that (i) $\Delta$ spans $V$. (ii)For $\alpha \in \Delta$, the root reflection $s_{\alpha}(h)=h-a_{h, \alpha} \alpha$, carry $\Delta$ to itself, where $h \in V, a_{h, \alpha}=\frac{2\langle h, \alpha>}{\langle\alpha, \alpha>}$. (iii) $a_{\beta, \alpha}$ is an integer whenever $\alpha, \beta \in \Delta$.

$l=\operatorname{dim} V$ is called the rank of $\Delta$. The Weyl group $W(\Delta)$ is the subgroup on orthogonal group of $V$ generated by the reflection $s_{\alpha}$ for $\alpha \in \Delta$. An abstract root system is said to be reduced if $\alpha \in \Delta$ implies $2 \alpha \notin \Delta$. If $\alpha$ is a root and $\frac{1}{2} \alpha$ is not a root, we say that $\alpha$ is reduced. An abstract root system $\Delta$ is said to be reducible if $\Delta$ admits an nontrivial disjoint decomposition $\Delta=\Delta^{\prime} \cup \Delta^{\prime \prime}$ with every member of $\Delta^{\prime}$ orthogonal to every member of $\Delta^{\prime \prime}$. We say $\Delta$ is irreducible if it admits no such nontrivial decomposition.

For $\alpha, \beta \in \Delta$, the $\alpha$ string containing $\beta$ is the set of all members of $\Delta \cup\{0\}$ of the form $\beta+k \alpha, k \in \mathbb{Z}$. In fact, there are no gaps, $-p \leq k \leq q, p \geq 0, q \geq 0$, and $a_{\beta, \alpha}=p-q$. The $\alpha$ string containing $\beta$ contains at most four roots.

We can choose a lexicographic ordering so that $\Delta=\Delta^{+} \cup \Delta^{-}$as disjoint sum of the set of positive roots and the set of negative roots. A root $\alpha$ is called simple if $\alpha>0$ and $\alpha$ does not decompose as the sum of two positive roots. A simple root is necessarily reduced. We can choose $l$ simple roots $\alpha_{1}, \ldots, \alpha_{l}$ which are linearly independent, every root $\alpha$ has the form $\alpha=\sum_{i=1}^{l} m_{i}(\alpha) \alpha_{i}$ with all $m_{i}(\alpha)$ nonnegative or nonpositive. We call $\Pi=\left\{\alpha_{1}, \cdots, \alpha_{l}\right\}$ a simple system or a fundamental system.

For an abstract root system we associate a Dynkin diagram.

Lemma 2.1. (classifications of root systems)[Hel,Kna]. Up to an isomorphism the irreducible reduced abstract root system are $A_{n}, B_{n}, C_{n}, D_{n}, E_{6}, E_{7}, E_{8}, F_{4}$ and $G_{2}$. For an nonreduced abstract root system, the reduced roots form an abstract reduced root system $\Delta_{s}$, the roots $\alpha \in \Delta$ such that $2 \alpha \notin \Delta$ form a reduced abstract root system $\Delta_{l}$, the Weyl group of $\Delta, \Delta_{s}, \Delta_{l}$ coincide. Up to an isomorphism the only irreducible abstract root system that are not reduced are of the form $(B C)_{n}$.

A subset $\Delta_{1}$ of $\Delta$ is called a subsystem of $\Delta$ if it is closed, i.e., (i)if $\alpha \in \Delta_{1}$, then $-\alpha \in \Delta_{1}$. (ii) if $\alpha, \beta \in \Delta_{1}, \alpha+\beta \in \Delta$, then $\alpha+\beta \in \Delta_{1}$. A subsystem $\Delta_{1}$ is called maximal if $\Delta_{1}$ is a proper subset of $\Delta$ and $\Delta_{1}$ is not properly contained in any properly subsystem of $\Delta$. A subsystem $\Delta_{1}$ is called $l-1$ maximal if it is maximal and $\operatorname{rank}\left(\Delta_{1}\right)=l-1$, where $l=\operatorname{rank}(\Delta)$.

Lemma 2.2. (classifications of l-1 maximal subsystems)[Wal]. (1)Let $\Delta$ be an irreducible reduced root system, the maximal (properly) subsystem is l maximal or l-1 maximal. (2)Let $\Delta_{1}$ be a l-1 maximal subsystem, $\Pi_{1}$ be a fundamental system of $\Delta_{1}$, then there exists a fundamental system $\Pi$ of $\Delta$ such that $\Pi_{1}=\Pi \cap \Delta_{1}$. (3)Let $\mu=\sum \mu_{i} \alpha_{i}$ be the highest root of $\Delta, \Delta_{1}=\left\{\alpha \in \Delta \mid \alpha=\sum m_{i} \alpha_{i}, m_{1}=0\right\}$ then $\Delta_{1}$ is l-1 maximal if and only if $\mu_{1}=1$. Every $l-1$ maximal subsystem can be obtained in this manner.

\section{REAL SEMISIMPLE LIE ALGEBRAS AND RESTRICTED ROOT SYSTEM}

We adopt the notation as in $[$ Hel]. Let $M=G / K$ be an irreducible Riemannian symmetric space of noncompact type with the Cartan decomposition $\mathfrak{g}=\mathfrak{k}+\mathfrak{p}$. We 
can extend the Cartan involution $\theta$ to be complex linear on the complex simple Lie algebra $\mathfrak{g}^{\mathbb{C}}$ :

$$
\left.\theta\right|_{\mathfrak{k}^{\mathbb{C}}}=i d,\left.\quad \theta\right|_{\mathfrak{p}^{\mathbb{C}}}=-i d .
$$

Up to isomorphism the real simple Lie algebra $\mathfrak{g}$ is uniquely determined by $\mathfrak{g}^{\mathbb{C}}$ and $\theta$.

Let $\mathfrak{h}=\mathfrak{h}_{\mathfrak{k}}+\mathfrak{a}$ be a Cartan subalgebra of $\mathfrak{g}$ with $\mathfrak{a}$ be maximal in $\mathfrak{p}$.

$$
\operatorname{dim} \mathfrak{a}=\operatorname{rank}(M)=r .
$$

Let $\Delta=\Delta\left(\mathfrak{g}^{\mathbb{C}}, \mathfrak{h}^{\mathbb{C}}\right)$ be the corresponding root system of $\mathfrak{g}^{\mathbb{C}}$.

Every root takes real value on $\mathfrak{h}_{\mathfrak{o}}=\mathfrak{i h}_{\mathfrak{k}}+\mathfrak{a}$. Through the Killing form of $\mathfrak{g}^{\mathbb{C}}$ we can embed the root into $\mathfrak{h}_{0}$ by

$$
\alpha(h)=B(\alpha, h), \quad \text { for } \quad h \in \mathfrak{h}^{\mathbb{C}} .
$$

The restricted roots are the elements in $\mathfrak{a}$ of the form

$$
\left\{\lambda=\alpha^{\prime}=\frac{1}{2}(\alpha-\theta \alpha) \mid a \in \Delta\right\} .
$$

All the restricted roots form an abstract root system $\Sigma$ which can be nonreduced. For $\lambda \in \Sigma$, we denote $\mathfrak{g}_{\lambda}$ the corresponding root space with multiplicity

$$
m_{\lambda}=\operatorname{dim} \mathfrak{g}_{\lambda} .
$$

We can choose the ordering in $\Sigma$ which is compatible to the ordering of $\Delta$.

The restricted roots and the restricted root spaces have the following properties:

(i) $\mathfrak{g}_{\mathfrak{o}}=\mathfrak{a} \oplus \mathfrak{m}, \mathfrak{m}=Z_{\mathfrak{k}}(\mathfrak{a})=\{Z \in \mathfrak{k} \mid[Z, A]=0 \quad$ for all $A \in \mathfrak{a}\}$. .

(ii) $\left[\mathfrak{g}_{\lambda}, \mathfrak{g}_{\mu}\right] \subset \mathfrak{g}_{\lambda+\mu}$.

(iii) $\theta\left(\mathfrak{g}_{\lambda}\right)=\mathfrak{g}_{-\lambda}$, and $\lambda \in \Sigma$ implies $-\lambda \in \Sigma$.

(iv) $\mathfrak{g}=\mathfrak{g}_{0}+\sum_{\lambda \in \Sigma} \mathfrak{g}_{\lambda}$ as direct sum.

For any $X \in \mathfrak{g}$, there exists $H \in \mathfrak{a}, X_{0} \in \mathfrak{m}, X_{\lambda} \in \mathfrak{g}_{\lambda}$ so that

$X=H+X_{0}+\sum_{\lambda \in \Sigma} X_{\lambda}=\left(X_{0}+\sum_{\lambda \in \Sigma^{+}}\left(X_{-\lambda}+\theta X_{-\lambda}\right)\right)+H+\sum_{\lambda \in \Sigma^{+}}\left(X_{\lambda}-\theta X_{-\lambda}\right)$.

We have Iwasawa decomposition $\mathfrak{g}=\mathfrak{k} \oplus \mathfrak{a} \oplus \sum_{\lambda \in \Sigma^{+}} \mathfrak{g}_{\lambda}$.

Let $h \in \mathfrak{a}$, the centralizer of $h$ in $\mathfrak{p}$ is

$$
Z_{\mathfrak{p}}(h)=\mathfrak{a}+\sum_{\lambda \in \Sigma^{+}, \lambda(h)=0} \mathfrak{g}_{\lambda} .
$$

Our objective is to get $s=\max _{h \in \mathfrak{a}}\left\{\operatorname{dim} Z_{\mathfrak{p}}(h)\right\}$.

$h \in \mathfrak{a}$ is called regular if $\lambda(h) \neq 0$ for all $\lambda \in \Sigma$, otherwise singular. Let $C^{+}=\left\{h \in \mathfrak{a} \mid \lambda(h)>0\right.$ for $\left.\lambda \in \Sigma^{+}\right\}$be a restricted Weyl chamber, its closure is the closed Weyl chamber $\overline{C^{+}}$. Let $W(\Sigma)$ be the Weyl group of $\Sigma$, we know for any $h \in \mathfrak{a}$, there exists $w \in W(\Sigma)$ such that $w(h) \in \overline{C^{+}}$.

Let $r=\operatorname{dim} \mathfrak{a}$ denote the $r a n k$ of M. If $h$ is regular then $\operatorname{dim} Z_{\mathfrak{p}}(h)=\operatorname{dim}(\mathfrak{a})=r$. Obviously if $\operatorname{dim} Z_{\mathfrak{p}}(h)$ takes maximal value $h$ must be a singular element. Now let $h$ be singular, set $\Sigma(h)=\{\lambda \in \Sigma \mid \lambda(h)=B(\lambda, h)=0\}$. It is obviously a subsystem of $\Sigma$. On the other hand, let $\Sigma_{1}$ is a subsystem of $\Sigma$ with $\operatorname{rank}\left(\Sigma_{1}\right)<r$, the span of $\Sigma_{1}$ is $V_{1}$, then $\operatorname{dim} V_{1}<r$, Let $h_{0}$ be any nonzero vector in the orthogonal complement of $V_{1}$ in $\mathfrak{a}$, then $\Sigma\left(h_{0}\right)$ is a subsystem contains $\Sigma_{1}$. If $\operatorname{rank}\left(\Sigma_{1}\right)=r$, the orthogonal complement of $\Sigma_{1}$ in $\mathfrak{a}$ is 0 , there exists no nonzero vector $h$ in $\mathfrak{a}$ 
with $\Sigma(h)$ contains $\Sigma_{1}$. So if $\operatorname{dim} \Sigma\left(h_{0}\right)$ takes the maximum $s, \Sigma\left(h_{0}\right)$ should be a maximal $l-1$ subsystem.

Let $\Pi^{\prime}=\left\{\lambda_{1}, \cdots, \lambda_{r}\right\}$ be the fundamental system of $\Sigma$. From the lemma of maximal subsystem we see that $l-1$ maximal subsystem must be of the form of $\Sigma_{k}=\left\{\lambda \in \Sigma \mid \lambda=\sum m_{i}^{\prime} \lambda_{i}, m_{k}^{\prime}=0\right\}$, where $1 \leq k \leq r$.

Since $s$ is the maximum of $\operatorname{dim} Z_{\mathfrak{p}}(h)$ as $h$ varies in $\mathfrak{a}$, from (3.2) we have

$$
s=r+\max _{1 \leq k \leq r} \sum_{\lambda \in \Sigma_{k}^{+}} m_{\lambda} .
$$

We note that $s=r$ if $r=1$. The restricted root systems and the Stake diagrams are listed in [Hel, p532-534].

\section{THE PARTIAL POSITIVITY FOR RIEMANNIAN SYMMETRIC SPACES}

Let $M=U / K$ be a simply connected irreducible symmetric spaces of compact type. Let $\mathfrak{u}=\mathfrak{k}+\mathfrak{p}_{*}$ be its Cartan decomposition. We identify $\mathfrak{p}_{*}$ with the tangent space of $M$ at $o=e K$. From the theory of symmetric spaces, up to constants there exists uniquely $U$-invariant Riemannian metric on $M$. We fix such one invariant metric. We know the curvature tensor at $o$ is

$$
R(X, Y) Z=-[[X, Y], Z], \quad \text { for all } X, Y, Z \in \mathfrak{p}_{*} .
$$

If $X, Y \in \mathfrak{p}_{*}$ are orthonormal vectors, then the sectional curvature of the plane spanned by $X$ and $Y$ is

$$
K(X, Y)=\|[X, Y]\|^{2} .
$$

Thus $K(X, Y)=0$ if and only if $[X, Y]=0$. Suppose the $M$ has s-positive curvature. From definition we have

(1)For any $s+1$ orthonormal vectors $\left\{X_{0}, X_{1}, \cdots, X_{s}\right\}$ in $\mathfrak{p}_{*}$

$$
\sum_{i=1}^{s} K\left(X_{0}, X_{i}\right)=\sum_{i=1}^{s}\left\|\left[X_{0}, X_{i}\right]\right\|^{2}>0 .
$$

(2)There exists $\mathrm{s}$ orthonormal vectors $\left\{X_{0}, X_{1}, \cdots, X_{s-1}\right\}$ in $\mathfrak{p}_{*}$

$$
\sum_{i=1}^{s-1} K\left(X_{0}, X_{i}\right)=\sum_{i=1}^{s-1}\left\|\left[X_{0}, X_{i}\right]\right\|^{2}=0
$$

This is equivalent to

(3)For any $X \in \mathfrak{p}_{*}, \operatorname{dim} Z_{\mathfrak{p}_{*}}(X)<s+1$, where

$$
Z_{\mathfrak{p}_{*}}(X)=\left\{Y \in \mathfrak{p}_{*} \mid[Y, X]=0\right\}
$$

is the centralizer of $X$ in $\mathfrak{p}_{*}$. And

(4)There exists at least $X_{0} \in \mathfrak{p}_{*}, \operatorname{dim} Z_{\mathfrak{p}_{*}}\left(X_{0}\right)=s$.

So we have

$$
s=\max _{X \in \mathfrak{p}_{*}}\left\{\operatorname{dim} Z_{\mathfrak{p}_{*}}(X)\right\} .
$$

We calculate the $\mathrm{s}$ in the following program:

Let $\Pi=\left\{\alpha_{1}, \cdots, \alpha_{l}\right\}$ be the simple system of the complex simple Lie algebra $\mathfrak{g}^{\mathbb{C}}$. Every root can be given as the integral linear combination

$$
\alpha=m_{1}(\alpha) \alpha_{1}+m_{2}(\alpha) \alpha_{2}+\cdots+m_{l}(\alpha) \alpha_{l} .
$$


For $\alpha \in \Delta, \alpha^{\prime}$ is its restriction ( or projection ) to $\mathfrak{a}$. We have

$$
\alpha_{i}^{\prime}=\lambda_{i^{\theta}}, 1 \leq i^{\theta} \leq r, \text { for } 1 \leq i \leq l
$$

Then

$$
\alpha^{\prime}=\sum_{i=1}^{l} m_{i} \lambda_{i^{\theta}}=\sum_{j=1}^{r} m_{j}^{\prime}(\alpha) \lambda_{j} .
$$

From this we can get all restricted roots and their multiplicities.

However, in our calculations, we need not know the multiplicities explicitly. From (3.3), for $1 \leq k \leq r$, let

$$
\Delta_{k}=\left\{\alpha \in \Delta \mid \alpha^{\prime} \neq 0, m_{k}^{\prime}(\alpha)=0\right\}
$$

we denote the number of positive roots in $\Delta(k)$ by $s_{k}^{\prime}$, let $s_{k}=r+s_{k}^{\prime}$. then we have

$$
s=\max _{1 \leq k \leq r}\left\{s_{k}\right\} .
$$

In the following calculation, we suppose that $r>1$ since $s=r=1$ if $r=1$. For four classical complex simple Lie algebras we imbed the root system into Euclidean spaces. We adopt the Dynkin diagrams of complex simple Lie algebras as in Hel] p476]. The root system $\Delta=\Delta\left(\mathfrak{g}^{\mathbb{C}}, \mathfrak{h}^{\mathbb{C}}\right)$ is reduced while the restricted root system $\Sigma=\Sigma(\mathfrak{g}, \mathfrak{a})$ may be non reduced. We denote the rank of $\Delta$ and $\Sigma$ by $l$ and $r$ respectively. Note that our l,r are r,l in the notation of [Hel p532-534]. For simple Lie algebra of exception type, we can get all the roots as listed in Frv.

The following formulas are trivial

$$
\begin{array}{r}
(l-k)(l-1-k)=l(l-1)+k^{2}-k(2 l-1) . \\
(l-k)(l-1-k)-(l-r)(l-1-r)=(r-k)(2 l-1-r-k) .
\end{array}
$$

Lemma 4.1. Let $f(t)=t(T-t), T>0, t$ takes integer values $1,2, \cdots, r, \quad r \leq T$, if $T-r \geq 1$, then

$$
\min _{1 \leq t \leq r} f(t)=f(1) .
$$

The Dynkin diagram of $\mathfrak{a}_{l}=\mathfrak{s l}(l+1, \mathbb{C})$ is

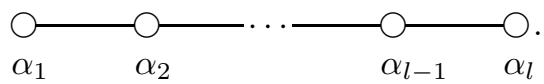

Let $\varepsilon_{j}, 1 \leq j \leq l+1$, be an orthogonal base of $\mathbb{R}^{l+1}$ with $\left|\varepsilon_{j}\right|^{2}=\frac{1}{2(l+1)}$. The simple root system of $\mathfrak{a}_{l}$ is

$$
\Pi=\left\{\alpha_{j}=\varepsilon_{j}-\varepsilon_{j+1}, j=1,2, \cdots, l\right\}, \quad\left|\alpha_{j}\right|^{2}=\frac{1}{l+1} .
$$

The positive roots are

$$
\varepsilon_{i}-\varepsilon_{j}=\alpha_{i}+\cdots+\alpha_{j-1}, 1 \leq i<j \leq l+1 .
$$

(1)AI, $\mathrm{M}=S U(n) / S O(n), \mathfrak{g}^{\mathbb{C}}=\mathfrak{a}_{l}=\mathfrak{s l}(n, \mathbb{C}), r=l=n-1$.

In this case, the restricted root system $\Sigma=\Delta$.

For $1 \leq k \leq r=l, \lambda_{k}=\alpha_{k}^{\prime}$, then $\alpha^{\prime}=\left(\sum m_{i} \alpha_{i}\right)^{\prime}=\sum m_{i} \lambda_{i}$.

We have

$$
\Delta_{k}^{+}=\left\{\alpha>0 \mid m_{k}(\alpha)=0\right\}
$$


Let $\alpha=\varepsilon_{i}-\varepsilon_{j}=\alpha_{i}+\cdots+\alpha_{j}, 1 \leq i<j \leq l+1, m_{k}(\alpha)=0$ if and only if

$$
i, j \leq k \text { or } i, j>k \text {. }
$$

Then

$$
\begin{aligned}
s_{k} & =r+\frac{k(k-1)}{2}+\frac{(l+1-k)(l-k)}{2} \\
& =-k(l+1-k)+r+\frac{(l+1) l}{2} .
\end{aligned}
$$

From Lemma 4.1 we get

$$
s=\max _{1 \leq k \leq r} s_{k}=s_{1}=l+\frac{l(l-1)}{2}=\frac{l(l+1)}{2}=\frac{n(n-1)}{2} .
$$

(2) AII, $M=S U(2 n) / S p(n), \mathfrak{g}^{\mathbb{C}}=\mathfrak{a}_{l}, l=2 n-1, r=n-1, l=2 r+1$.

We have

$$
\alpha_{1}^{\prime}=\alpha_{3}^{\prime}=\cdots=\alpha_{2 r-1}^{\prime}=\alpha_{2 r+1}^{\prime}=0 ; \quad \alpha_{2}^{\prime}=\lambda_{1}, \alpha_{4}^{\prime}=\lambda_{2}, \cdots, \alpha_{2 r}^{\prime}=\lambda_{r} .
$$

Let $\alpha=\varepsilon_{i}-\varepsilon_{j}, 1 \leq 1<j \leq l+1=2 n, \alpha^{\prime}=0$ if and only if $\alpha=\alpha_{1}, \alpha_{3}, \cdots, \alpha_{2 n-1}$.

For $1 \leq k \leq r=n-1, \Delta_{k}=\left\{\alpha \in \Delta \mid m_{k}^{\prime}(\alpha)=0\right\}=\left\{\alpha \in \Delta \mid m_{2 k}^{\prime}(\alpha)=0\right\}$, and

$$
m_{2 k}(\alpha)=0 \text { if and only if } i, j \leq 2 k \text { or } j>i>2 k .
$$

We see that

$$
\begin{aligned}
s_{k} & =r+\frac{2 k(2 k-1)}{2}+\frac{(l+1-2 k)(l-2 k)}{2}-(r+1) \\
& =-2 k(l+1-2 k)+\frac{(l+1) l}{2}-1 .
\end{aligned}
$$

From Lemma 4.1 we get

$$
s=s_{1}=\frac{(l-1)(l-2)}{2}=(n-1)(2 n-3) .
$$

(3) AIII, we suppose that $p \leq q, M=S U(p+q) / S\left(U_{p} \times U_{q}\right), \mathfrak{g}=\mathfrak{a}_{l}, l=$ $p+q-1, r=\min (p, q)=p, r \leq \frac{l+1}{2}$.

We have

$$
\alpha_{i}^{\prime}=\alpha_{l+1-i}^{\prime}=\lambda_{i}, 1 \leq i \leq r ; \quad \alpha_{j}^{\prime}=0, r<j \leq l-r .
$$

Let $\alpha=\varepsilon_{i}-\varepsilon_{j}, 1 \leq i<j \leq l+1, \alpha^{\prime}=0$ if and only if $r<i<j \leq l+1-r$. $m_{k}^{\prime}(\alpha)=0$ if $m_{k}(\alpha)=0$ or $m_{l+1-k}(\alpha)=0$.

Then $m_{k}^{\prime}(\alpha)=0$ implies

$$
1 \leq i<j \leq k \text { or } l+1-k<i<j \leq l+1 \text { or } k \leq i<j \leq l+1-k .
$$

We get

$$
\begin{aligned}
s_{k} & =r+2 \frac{k(k-1)}{2}+\frac{(l+1-2 k)(l-2 k)}{2}-\frac{(l+1-2 r)(l-2 r)}{2} \\
& =k(k-1)+2 k^{2}-k(2 l+1)+\frac{(l+1) l}{2}+r-\frac{(l+1-2 r)(l-2 r)}{2} \\
& =-k(2 l+2-3 k)+\frac{(l+1) l}{2}+r-\frac{(l+1-2 r)(l-2 r)}{2} .
\end{aligned}
$$

If $\frac{2 l+2}{3}-r \geq 1$, ie., $r \leq \frac{2 l-1}{3}$ then $\max \left\{s_{k}\right\}=s_{1}$.

As $r \leq \frac{l+1}{2}$, we have $r \leq \frac{2 l-1}{3}$ when $l \geq 5$. 
we have for $l \geq 5$, i.e., $q \geq 3$,

$$
\begin{aligned}
s=s_{1} & =r+\frac{(l+1-2)(l-2)}{2}-\frac{(l+1-2 r)(l-2 r)}{2} \\
& =r+\frac{1}{2}(2 r-2)(2 l+1-2 r-2) \\
& =1+(r-1)+(r-1)(2 l-1-2 r) \\
& =1+2(r-1)(l-r) \\
& =1+2(p-1)(q-1) .
\end{aligned}
$$

If $l=4, r \leq \frac{5}{2}, r=2($ we suppose $r \geq 2)$, as $1 \cdot(10-3)<2 \cdot(10-6)$, thens $s_{1}>$ $s_{2}, s=s_{2}$. In this case $p=2, q=3$.

If $l=3, r \leq 2, r=2$, as $1 \cdot(8-3)>2 \cdot(8-6)$, then $s_{1}<s_{2}, s=s_{2}=4$. In this case $p=q=2$.

The Dynkin diagram of $\mathfrak{b}_{l}=\mathfrak{s o}(2 l+1, \mathbb{C})$ is

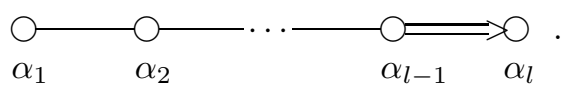

Let $\varepsilon_{j}, 1 \leq j \leq l$, be an orthogonal base of $\mathbb{R}^{l},\left|\varepsilon_{j}\right|^{2}=\frac{1}{2(2 l-1)}$, the simple root system of $\mathfrak{b}_{l}$ is

$$
\Pi=\left\{\alpha_{j}=\varepsilon_{j}-\varepsilon_{j+1}, j=1,2, \cdots, l-1, \alpha_{l}=\varepsilon_{l}\right\} .
$$

The positive roots are

$$
\varepsilon_{i}, \varepsilon_{i} \pm \varepsilon_{j}, 1 \leq i<j \leq l \text {. }
$$

where

$$
\begin{gathered}
\varepsilon_{i}=\alpha_{i}+\cdots+\alpha_{l} \\
\varepsilon_{i}-\varepsilon_{j}=\alpha_{i}+\cdots+\alpha_{j} \\
\varepsilon_{i}+\varepsilon_{j}=\alpha_{i}+\cdots+\alpha_{j-1}+2\left(\alpha_{j}+\cdots+\alpha_{l}\right) .
\end{gathered}
$$

(4)BI, $M=S O(p+q) / S O(p) \times S O(q), p+q=2 l+1, \mathfrak{g}=\mathfrak{b}_{l}, r=p \leq l$.

We have

$$
\alpha_{i}^{\prime}=\lambda_{i}, 1 \leq i \leq r ; \quad \alpha_{j}^{\prime}=0, r<j \leq l .
$$

If $\alpha \in \Delta^{+}, \alpha^{\prime}=0$ then $\alpha$ is one of the following roots,

$$
\varepsilon_{i}, i>r ; \quad \varepsilon_{i} \pm \varepsilon_{j}, j>i>r .
$$

If $\alpha>0, m_{k}^{\prime}(\alpha)=0$, then $\alpha$ is one of the following roots,

$$
\varepsilon_{i}, i>k ; \quad \varepsilon_{i}-\varepsilon_{j}, i<j \leq k \text { or } j>i>k ; \quad \varepsilon_{i}+\varepsilon_{j}, j>i>k .
$$

Then

$$
\begin{aligned}
s_{k} & =r+[(l-k)-(l-r)]+\frac{k(k-1)}{2}+2\left[\frac{(l-k)(l-1-k)}{2}-\frac{(l-r)(l-1-r)}{2}\right] \\
& =2 r-k+\frac{k(k-1)}{2}+k^{2}-k(2 l-1)+l(l-1)-(l-r)(l-1-r) \\
& =-\frac{k}{2}(4 l+1-3 k)+2 r+l(l-1)-(l-r)(l-1-r) .
\end{aligned}
$$


Since $\frac{4 l+1}{3}-r \geq \frac{4 l+1}{3}-l=\frac{l+1}{3} \geq 1$, we have

$$
\begin{aligned}
s=s_{1} & =2 r-1+(l-1)(l-2)-(l-r)(l-1-r) \\
& =1+2(r-1)+(r-1)(2 l-1-r-1) \\
& =1+(r-1)(2 l-r) \\
& =1+(p-1)(q-1)
\end{aligned}
$$

The Dynkin diagram of $\mathfrak{d}_{l}=\mathfrak{s o}(2 l, \mathbb{C})$ is

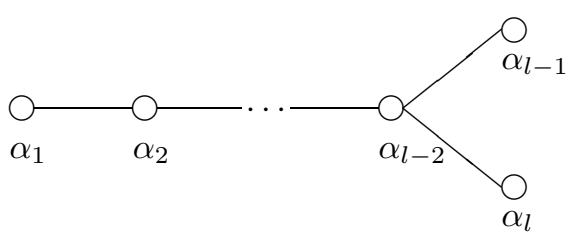

Let $\varepsilon_{j}, 1 \leq j \leq l$, be an orthogonal base of $\mathbb{R}^{l},\left|\varepsilon_{j}\right|^{2}=\frac{1}{4(l-1)}$. The simple root system is

$$
\Pi=\left\{\alpha_{j}=\varepsilon_{j}-\varepsilon_{j+1}, j=1,2, \cdots, l-1, \alpha_{l}=\varepsilon_{l-1}+\varepsilon_{l}\right\} .
$$

The positive roots are

$$
\varepsilon_{i} \pm \varepsilon_{j}, i<j .
$$

where

$$
\begin{aligned}
\varepsilon_{i}-\varepsilon_{j} & =\alpha_{i}+\cdots+\alpha_{j-1} \\
\varepsilon_{i}+\varepsilon_{j} & =\alpha_{i}+\cdots+\alpha_{l-2}+\alpha_{j}+\cdots+\alpha_{l} \\
& =\alpha_{i}+\cdots+\alpha_{j-1}+2\left(\alpha_{j}+\cdots+\alpha_{l-2}\right)+\alpha_{l-1}+\alpha_{l} .
\end{aligned}
$$

(5)DI, $M=S O(p+q) / S O(p) \times S O(q), p+q=2 l, \mathfrak{g}=\mathfrak{d}_{l}, r=p \leq l$.

There are three cases.

(i) $2 \leq r \leq l-2$, we have

$$
\alpha_{i}^{\prime}=\lambda_{i}, 1 \leq i \leq r ; \quad \alpha_{j}^{\prime}=0, r<j \leq l .
$$

If $\alpha \in \Delta^{+}, \alpha^{\prime}=0$ then $\alpha$ is one of the following roots,

$$
\varepsilon_{i}, i>r ; \quad \varepsilon_{i} \pm \varepsilon_{j}, j>i>r .
$$

If $\alpha>0, m_{k}^{\prime}(\alpha)=0$, then $\alpha$ is one of the following roots,

$$
\varepsilon_{i}, i>k ; \quad \varepsilon_{i}-\varepsilon_{j}, i<j \leq k \text { or } j>i>k ; \quad \varepsilon_{i}+\varepsilon_{j}, j>i>k .
$$

Then

$$
\begin{aligned}
s_{k} & =r+\frac{k(k-1)}{2}+2\left[\frac{(l-k)(l-1-k)}{2}-\frac{(l-r)(l-1-r)}{2}\right] \\
& =\frac{k(k-1)}{2}+k^{2}-k(2 l-1)+l(l-1)-(l-r)(l-1-r)+r \\
& =-\frac{k}{2}(4 l-1-3 k)+l(l-1)-(l-r)(l-1-r)+r .
\end{aligned}
$$


Since $\frac{4 l-1}{3}-r \geq \frac{l+5}{3} \geq 1$, we have

$$
\begin{aligned}
s=s_{1} & =r+(l-1)(l-2)-(l-r)(l-1-r) \\
& =1+(r-1)+(r-1)(2 l-1-r-1) \\
& =1+(r-1)(2 l-1-r) \\
& =1+(p-1)(q-1) .
\end{aligned}
$$

(ii) $r=p=l-1, q=l+1$, we have

$$
\alpha_{i}^{\prime}=\lambda_{i}, 1 \leq i \leq l-2 ; \alpha_{l-1}^{\prime}=\alpha_{l}^{\prime}=\lambda_{l-1}=\lambda_{r} .
$$

$\alpha^{\prime}>0$ implies $\alpha=0$.

If $\alpha>0, m_{k}^{\prime}(\alpha)=0$, then $\alpha$ is one of the following roots,

$$
\varepsilon_{i}, i>k ; \quad \varepsilon_{i}-\varepsilon_{j}, i<j \leq k \text { or } j>i>k ; \quad \varepsilon_{i}+\varepsilon_{j}, j>i>k .
$$

Then

$$
\begin{aligned}
s_{k} & =r+\frac{k(k-1)}{2}+2 \frac{(l-k)(l-1-k)}{2} \\
& =\frac{k(k-1)}{2}+k^{2}-k(2 l-1)+l(l-1)+r \\
& =-\frac{k}{2}(4 l-1-3 k)+l(l-1)+r .
\end{aligned}
$$

Since $\frac{4 l-1}{3}-r=\frac{l+2}{3} \geq 1$, we have

$$
\begin{aligned}
s=s_{1} & =r+(l-1)(l-2) \\
& =(l-1)^{2} \\
& =1+l(l-2) \\
& =1+(p-1)(q-1) .
\end{aligned}
$$

(iii) $r=p=l, q=l$, we have

$$
\alpha_{i}^{\prime}=\lambda_{i}, 1 \leq i \leq l
$$

$\alpha^{\prime}>0$ implies $\alpha=0$.

If $\alpha>0, m_{k}^{\prime}(\alpha)=0$, then $\alpha$ is one of the following roots,

$$
\varepsilon_{i}, i>k ; \quad \varepsilon_{i}-\varepsilon_{j}, i<j \leq k \text { or } j>i>k ; \quad \varepsilon_{i}+\varepsilon_{j}, j>i>k .
$$

Then

$$
\begin{aligned}
s_{k} & =r+\frac{k(k-1)}{2}+2 \frac{(l-k)(l-1-k)}{2} \\
& =\frac{k(k-1)}{2}+k^{2}-k(2 l-1)+l(l-1)+r \\
& =-\frac{k}{2}(4 l-1-3 k)+l(l-1)+r .
\end{aligned}
$$

Since $\frac{4 l-1}{3}-r=\frac{l-1}{3}$, we have for $l \geq 4$, i.e., $q \geq 4$,

$$
\begin{aligned}
s=s_{1} & =r+(l-1)(l-2) \\
& =1+(l-1)+(l-1)(l-2) \\
& =1+(l-1)^{2} \\
& =1+(p-1)(q-1) .
\end{aligned}
$$

When $r \geq 2, l \geq r=2$. For $l=2, r=p=2, q=2, s_{1}<s_{2}, s=s_{2}=3$. 
For $l=3, r=p=2, q=4, s_{1}>s_{2}, s=s_{1} ; r=p=3, q=3, s_{3}>s_{1}>s_{2}$, $s=s_{3}=6$.

(6)DIII, $M=S O(2 n) / U(n), \mathfrak{g}^{\mathbb{C}}=\mathfrak{g}_{l}, l=n, r=\left[\frac{l}{2}\right]$. There are two cases.

(i) $l$ is even, we have

$$
\alpha_{2 i}^{\prime}=\lambda_{i}, 1 \leq i \leq r=\frac{l}{2} ; \alpha_{2 i-1}^{\prime}=0,1 \leq i \leq r .
$$

$\alpha>0, \alpha^{\prime}>0$ implies $\alpha=\alpha_{1}, \alpha_{3}, \cdots, \alpha_{l-1}$.

If $\alpha>0, m_{k}^{\prime}(\alpha)=0$, then $\alpha$ is one of the following roots,

$$
\varepsilon_{i}-\varepsilon_{j}, i<j \leq 2 k \text { or } j>i>2 k ; \quad \varepsilon_{i}+\varepsilon_{j}, j>i>2 k .
$$

Then

$$
\begin{aligned}
s_{k} & =r+\left[\frac{2 k(2 k-1)}{2}+\frac{(l-2 k)(l-1-2 k)}{2}-\frac{l}{2}\right]+\frac{(l-2 k)(l-1-2 k)}{2} \\
& =\frac{k(2 k-1)}{2}+(l-2 k)(l-1-2 k) \\
& =-k(4 l-1-6 k)+l(l-1) .
\end{aligned}
$$

For $\frac{4 l-1}{6}-\frac{l}{2}=\frac{l-1}{6} \geq 1$, i.e., $l \geq 7$, we have

$$
s=s_{1}=1+(l-2)(l-3)=1+(n-2)(n-3) .
$$

For $l=4, r=2, s_{1}<s_{2}, s=s_{2}=6$.

For $l=6, r=3, s_{2}<s_{1}<s_{3}, s=s_{3}=15$.

(ii) $l$ is odd, we have

$$
\alpha_{2 i}^{\prime}=\lambda_{i}, 1 \leq i \leq \frac{l-3}{2} ; \alpha_{l-1}^{\prime}=\alpha_{l}^{\prime}=\lambda_{r}, r=\frac{l-1}{2} ; \alpha_{2 i-1}^{\prime}=0,1 \leq i \leq r .
$$

$\alpha>0, \alpha^{\prime}>0$ implies $\alpha=\alpha_{1}, \alpha_{3}, \cdots, \alpha_{l-2}$.

If $\alpha>0, m_{k}^{\prime}(\alpha)=0$, then $\alpha$ is one of the following roots,

$$
\varepsilon_{i}-\varepsilon_{j}, i<j \leq 2 k \text { or } j>i>2 k ; \quad \varepsilon_{i}+\varepsilon_{j}, j>i>2 k .
$$

Then

$$
\begin{aligned}
s_{k} & =r+\left[\frac{2 k(2 k-1)}{2}+\frac{(l-2 k)(l-1-2 k)}{2}-r\right]+\frac{(l-2 k)(l-1-2 k)}{2} \\
& =\frac{k(2 k-1)}{2}+(l-2 k)(l-1-2 k) \\
& =-k(4 l-1-6 k)+l(l-1) .
\end{aligned}
$$

For $\frac{4 l-1}{6}-\frac{l-1}{2}=\frac{l+2}{6} \geq 1$, i.e., $l \geq 4$, we have

$$
s=s_{1}=1+(l-2)(l-3)=1+(n-2)(n-3) .
$$

For $l=3, r=1, s=r=1$.

The Dynkin diagram of $\mathfrak{c}_{l}=\mathfrak{s p}(l, \mathbb{C})$ is

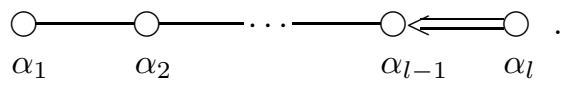

Let $\varepsilon_{j}, 1 \leq j \leq l$, be an orthogonal base of $\mathbb{R}^{l},\left|\varepsilon_{j}\right|^{2}=\frac{1}{4(l+1)}$, the simple root system is

$$
\Pi=\left\{\alpha_{j}=\varepsilon_{j}-\varepsilon_{j+1}, j=1,2, \cdots, l-1, \alpha_{l}=2 \varepsilon_{l}\right\} .
$$


The positive roots are

$$
2 \varepsilon_{i}, \varepsilon_{i} \pm \varepsilon_{j}, i<j
$$

where

$$
\begin{aligned}
2 \varepsilon_{i}= & 2\left(\alpha_{i}+\cdots+\alpha_{l-1}\right)+\alpha_{l} \\
\varepsilon_{i}-\varepsilon_{j}= & \alpha_{i}+\cdots+\alpha_{j-1} \\
\varepsilon_{i}+\varepsilon_{j}= & \alpha_{i}+\cdots+\alpha_{j-2}+2\left(\alpha_{j}+\cdots+\alpha_{l-1}\right)+\alpha_{l} .
\end{aligned}
$$

(7)CI, $M=S p(n) / U(n), \mathfrak{g}^{\mathbb{C}}=\mathfrak{c}_{l}, l=n$.

we have

$$
\alpha_{i}^{\prime}=\lambda_{i}, 1 \leq i \leq l
$$

$\alpha^{\prime}>0$ implies $\alpha=0$.

If $\alpha>0, m_{k}^{\prime}(\alpha)=0$, then $\alpha$ is one of the following roots,

$$
2 \varepsilon_{i}, i>k ; \quad \varepsilon_{i}-\varepsilon_{j}, i<j \leq 2 k \text { or } j>i>2 k ; \quad \varepsilon_{i}+\varepsilon_{j}, j>i>2 k .
$$

Then

$$
\begin{aligned}
s_{k} & =r+(l-k)+\frac{k(k-1)}{2}+2 \frac{(l-k)(l-1-k)}{2} \\
& =2 l-k+\frac{k(k-1)}{2}+(l-k)(l-1-k) \\
& =-k(4 l+1-3 k)+l(l-1)+2 l .
\end{aligned}
$$

For $\frac{4 l+1}{3}-l=\frac{l+1}{3} \geq 1$,i.e., $l \geq 2$, we have

$$
s=s_{1}=2 l-1+(l-1)(l-2)=1+l(l-1)=1+n(n-1) .
$$

(8)CII, $M=S p(p+q) / S P(p) \times S P(q), \mathfrak{g}^{\mathbb{C}}=\mathfrak{c}_{l}, l=p+q, r=p \leq\left[\frac{l}{2}\right]$.

We have

$$
\alpha_{2 i}^{\prime}=\lambda_{i}, 1 \leq i \leq r ; \alpha_{j}^{\prime}=0, j=1,3, \cdots, 2 r-1, \text { or } j>2 r .
$$

$\alpha>0, \alpha^{\prime}>0$ implies $\alpha=\alpha_{1}, \alpha_{3}, \cdots, \alpha_{2 r-1}$; or $\alpha=\varepsilon_{i}-\varepsilon_{j}, j>i>2 r$.

If $\alpha>0, m_{k}^{\prime}(\alpha)=0$, then $\alpha$ is one of the following roots,

$$
2 \varepsilon_{i}, i>2 k ; \quad \varepsilon_{i}-\varepsilon_{j}, i<j \leq 2 k \text { or } j>i>2 k ; \quad \varepsilon_{i}+\varepsilon_{j}, j>i>2 k .
$$

Then

$$
\begin{aligned}
s_{k} & =r+(r-k)+\left[\frac{2 k(2 k-1)}{2}-k\right]+2\left[\frac{(l-2 k)(l-1-2 k)}{2}-\frac{(l-2 r)(l-1-2 r)}{2}\right] \\
& =2 r+k(2 k-3)+(l-2 k)(l-1-2 k)-(l-2 r)(l-1-2 r) \\
& =-k(4 l+1-6 k)+2 r+l(l-1)-(l-2 r)(l-1-2 r) .
\end{aligned}
$$

For $\frac{4 l+1}{6}-\frac{l}{2}=\frac{l+1}{6} \geq 1$,i.e., $l \geq 5$, we have

$$
\begin{aligned}
s=s_{1} & =2 r-1+(l-2)(l-3)-(l-2 r)(l-1-2 r) \\
& =1+4(r-1)(l-1-r) \\
& =1+4(p-1)(q-1) .
\end{aligned}
$$

For $l=4, r=p=2, q=2, s_{1}<s_{2}, s=s_{2}=6$.

Now we consider the cases of exception type.

We draw the Dynkin diagram of $\mathfrak{e}_{6}$ as follows. 


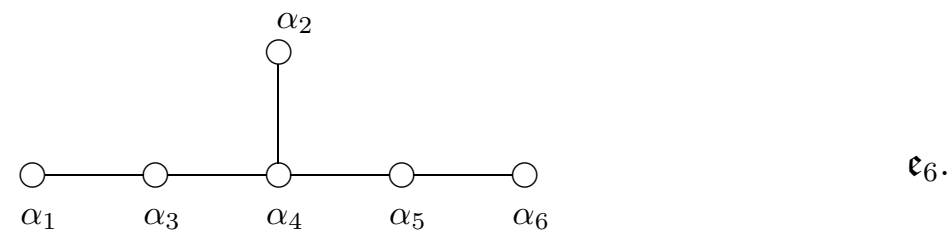

We list the positive roots of $\mathfrak{e}_{6}$ as follows:

$$
\begin{aligned}
& \alpha_{1}, \quad \alpha_{2}, \quad \alpha_{3}, \quad \alpha_{4}, \quad \alpha_{5}, \quad \alpha_{6} \\
& \alpha_{1}+\alpha_{3}, \quad \alpha_{3}+\alpha_{4}, \quad \alpha_{2}+\alpha_{4}, \quad \alpha_{4}+\alpha_{5}, \quad \alpha_{5}+\alpha_{6} \\
& \alpha_{1}+\alpha_{3}+\alpha_{4}, \quad \alpha_{3}+\alpha_{4}+\alpha_{2}, \quad \alpha_{3}+\alpha_{4}+\alpha_{5} \\
& \alpha_{4}+\alpha_{5}+\alpha_{2}, \quad \alpha_{4}+\alpha_{5}+\alpha_{6} \\
& \alpha_{1}+\alpha_{3}+\alpha_{4}+\alpha_{2}, \quad \alpha_{1}+\alpha_{3}+\alpha_{4}+\alpha_{5}, \quad \alpha_{2}+\alpha_{3}+\alpha_{4}+\alpha_{5} \\
& \alpha_{3}+\alpha_{4}+\alpha_{5}+\alpha_{6}, \quad \alpha_{2}+\alpha_{4}+\alpha_{5}+\alpha_{6} \\
& \alpha_{1}+\alpha_{2}+\alpha_{3}+\alpha_{4}+\alpha_{5}, \quad \alpha_{1}+\alpha_{3}+\alpha_{4}+\alpha_{5}+\alpha_{6} \\
& \alpha_{2}+\alpha_{3}+\alpha_{4}+\alpha_{5}+\alpha_{6}, \quad \alpha_{2}+\alpha_{3}+2 \alpha_{4}+\alpha_{5} \\
& \alpha_{1}+\alpha_{2}+\alpha_{3}+\alpha_{4}+\alpha_{5}+\alpha_{6}, \quad \alpha_{1}+\alpha_{2}+\alpha_{3}+2 \alpha_{4}+\alpha_{5} \\
& \alpha_{2}+\alpha_{3}+2 \alpha_{4}+\alpha_{5}+\alpha_{6} \\
& \alpha_{1}+\alpha_{2}+\alpha_{3}+2 \alpha_{4}+\alpha_{5}+\alpha_{6}, \quad \alpha_{1}+\alpha_{2}+2 \alpha_{3}+2 \alpha_{4}+\alpha_{5} \\
& \alpha_{2}+\alpha_{3}+2 \alpha_{4}+2 \alpha_{5}+\alpha_{6} \\
& \alpha_{1}+\alpha_{2}+2 \alpha_{3}+2 \alpha_{4}+\alpha_{5}+\alpha_{6}, \quad \alpha_{1}+\alpha_{2}+\alpha_{3}+2 \alpha_{4}+2 \alpha_{5}+\alpha_{6} \\
& \alpha_{1}+\alpha_{2}+2 \alpha_{3}+2 \alpha_{4}+2 \alpha_{5}+\alpha_{6} \\
& \alpha_{1}+\alpha_{2}+2 \alpha_{3}+3 \alpha_{4}+2 \alpha_{5}+\alpha_{6} \\
& \alpha_{1}+2 \alpha_{2}+2 \alpha_{3}+3 \alpha_{4}+2 \alpha_{5}+\alpha_{6} .
\end{aligned}
$$

$(9) \mathrm{EI}, \mathfrak{g}^{\mathbb{C}}=\mathfrak{e}_{l}, l=6, r=6$.

we have

$$
\alpha_{i}^{\prime}=\lambda_{i}, 1 \leq i \leq 6 .
$$

$\alpha^{\prime}>0$ implies $\alpha=0$.

If $\alpha>0, m_{k}^{\prime}(\alpha)=0$, then $m_{k}(\alpha)=0$.

We list $\Delta_{k}^{+}$as follows,

$$
\begin{aligned}
& \Delta_{1}^{+}=\left\{\alpha_{3}, \quad \alpha_{4}, \quad \alpha_{5}, \quad \alpha_{6}, \quad \alpha_{2},\right. \\
& \alpha_{3}+\alpha_{4}, \quad \alpha_{4}+\alpha_{2}, \quad \alpha_{4}+\alpha_{5}, \quad \alpha_{5}+\alpha_{6}, \\
& \alpha_{3}+\alpha_{4}+\alpha_{2}, \quad \alpha_{3}+\alpha_{4}+\alpha_{5}, \quad \alpha_{4}+\alpha_{5}+\alpha_{2}, \quad \alpha_{4}+\alpha_{5}+\alpha_{6}, \\
& \alpha_{3}+\alpha_{4}+\alpha_{5}+\alpha_{2}, \quad \alpha_{3}+\alpha_{4}+\alpha_{5}+\alpha_{6}, \quad \alpha_{4}+\alpha_{5}+\alpha_{6}+\alpha_{2}, \\
& \alpha_{3}+\alpha_{4}+\alpha_{5}+\alpha_{6}+\alpha_{2}, \quad \alpha_{3}+2 \alpha_{4}+\alpha_{5}+\alpha_{2}, \\
& \left.\alpha_{3}+2 \alpha_{4}+\alpha_{5}+\alpha_{6}+\alpha_{2}, \alpha_{3}+2 \alpha_{4}+2 \alpha_{5}+\alpha_{6}+\alpha_{2}\right\} \\
& \Delta_{2}^{+}=\left\{\begin{array}{lllll}
\alpha_{1}, & \alpha_{3}, & \alpha_{4}, & \alpha_{5}, & \alpha_{6}
\end{array},\right. \\
& \alpha_{1}+\alpha_{3}, \quad \alpha_{3}+\alpha_{4}, \quad \alpha_{4}+\alpha_{5}, \quad \alpha_{5}+\alpha_{6}, \\
& \alpha_{1}+\alpha_{3}+\alpha_{4}, \quad \alpha_{3}+\alpha_{4}+\alpha_{5}, \quad \alpha_{4}+\alpha_{5}+\alpha_{6}, \\
& \alpha_{1}+\alpha_{3}+\alpha_{4}+\alpha_{5}, \quad \alpha_{3}+\alpha_{4}+\alpha_{5}+\alpha_{6}, \\
& \left.\alpha_{1}+\alpha_{3}+\alpha_{4}+\alpha_{5}+\alpha_{6}\right\}
\end{aligned}
$$




$$
\begin{aligned}
& \Delta_{3}^{+}=\left\{\alpha_{1}, \quad \alpha_{4}, \quad \alpha_{5}, \quad \alpha_{6}, \quad \alpha_{2}, \alpha_{4}+\alpha_{2}, \quad \alpha_{4}+\alpha_{5}, \quad \alpha_{5}+\alpha_{6},\right. \\
& \left.\alpha_{4}+\alpha_{5}+\alpha_{2}, \quad \alpha_{4}+\alpha_{5}+\alpha_{6}, \quad \alpha_{4}+\alpha_{5}+\alpha_{6}+\alpha_{2}\right\}
\end{aligned}
$$

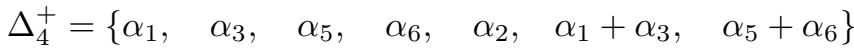

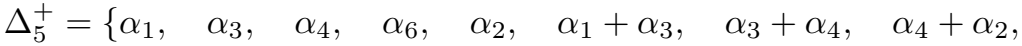

$$
\begin{aligned}
& \left.\alpha_{1}+\alpha_{3}+\alpha_{4}, \quad \alpha_{3}+\alpha_{4}+\alpha_{2}, \quad \alpha_{1}+\alpha_{3}+\alpha_{4}+\alpha_{2}\right\} \\
& \Delta_{6}^{+}=\left\{\alpha_{1}, \quad \alpha_{3}, \quad \alpha_{4}, \quad \alpha_{5}, \quad \alpha_{2},\right. \\
& \alpha_{1}+\alpha_{3}, \quad \alpha_{3}+\alpha_{4}, \quad \alpha_{4}+\alpha_{2}, \quad \alpha_{4}+\alpha_{5}, \\
& \alpha_{1}+\alpha_{3}+\alpha_{4}, \quad \alpha_{3}+\alpha_{4}+\alpha_{2}, \quad \alpha_{3}+\alpha_{4}+\alpha_{5}, \quad \alpha_{4}+\alpha_{5}+\alpha_{2}, \quad \alpha_{1}+\alpha_{3}+\alpha_{4}+\alpha_{2}, \\
& \alpha_{1}+\alpha_{3}+\alpha_{4}+\alpha_{5}, \quad \alpha_{3}+\alpha_{4}+\alpha_{5}+\alpha_{2}, \\
& \alpha_{1}+\alpha_{3}+\alpha_{4}+\alpha_{5}+\alpha_{2}, \quad \alpha_{3}+2 \alpha_{4}+\alpha_{5}+\alpha_{2}, \\
& \left.\alpha_{1}+\alpha_{3}+2 \alpha_{4}+\alpha_{5}+\alpha_{2}, \quad \alpha_{1}+2 \alpha_{3}+2 \alpha_{4}+\alpha_{5}+\alpha_{2}\right\} \text {. }
\end{aligned}
$$

We get the $\mathrm{s}$ values as follow

$$
s_{1}=26, s_{2}=21, s_{3}=17, s_{4}=13, s_{5}=17, s_{6}=26 \text {. }
$$

Then $s=26$.

(10)EII, $\mathfrak{g}^{\mathbb{C}}=\mathfrak{e}_{l}, l=6, r=4$.

we have

$$
\alpha_{1}^{\prime}=\alpha_{6}^{\prime}=\lambda_{1}, \alpha_{3}^{\prime}=\alpha_{5}^{\prime}=\lambda_{3}, \alpha_{2}^{\prime}=\lambda_{2}, \alpha_{4}^{\prime}=\lambda_{4} .
$$

$\alpha^{\prime}=0$ implies $\alpha=0$.

We list $\Delta_{k}^{+}$as follows,

$$
\begin{aligned}
& \Delta_{1}^{+}=\left\{\alpha_{3}, \quad \alpha_{4}, \quad \alpha_{5}, \quad \alpha_{2},\right. \\
& \alpha_{3}+\alpha_{4}, \quad \alpha_{4}+\alpha_{2}, \quad \alpha_{4}+\alpha_{5}, \\
& \alpha_{3}+\alpha_{4}+\alpha_{2}, \quad \alpha_{3}+\alpha_{4}+\alpha_{5}, \quad \alpha_{4}+\alpha_{5}+\alpha_{2}, \\
& \left.\alpha_{3}+\alpha_{4}+\alpha_{5}+\alpha_{2}, \quad \alpha_{3}+2 \alpha_{4}+\alpha_{5}+\alpha_{2}\right\} \\
& \Delta_{2}^{+}=\left\{\alpha_{1}, \quad \alpha_{3}, \quad \alpha_{4}, \quad \alpha_{5}, \quad \alpha_{6},\right. \\
& \alpha_{1}+\alpha_{3}, \quad \alpha_{3}+\alpha_{4}, \quad \alpha_{4}+\alpha_{5}, \quad \alpha_{5}+\alpha_{6}, \\
& \alpha_{1}+\alpha_{3}+\alpha_{4}, \quad \alpha_{3}+\alpha_{4}+\alpha_{5}, \quad \alpha_{4}+\alpha_{5}+\alpha_{6}, \\
& \alpha_{1}+\alpha_{3}+\alpha_{4}+\alpha_{5}, \quad \alpha_{3}+\alpha_{4}+\alpha_{5}+\alpha_{6}, \\
& \left.\alpha_{1}+\alpha_{3}+\alpha_{4}+\alpha_{5}+\alpha_{6}\right\}
\end{aligned}
$$

We get $s_{k}$ as follows,

$$
s_{1}=16, s_{2}=19, s_{3}=9, s_{4}=11 .
$$

Then $s=19$.

(11)EIII, $\mathfrak{g}^{\mathbb{C}}=\mathfrak{e}_{l}, l=6, r=2$.

we have

$$
\alpha_{1}^{\prime}=\alpha_{6}^{\prime}=\lambda_{1}, \alpha_{2}^{\prime}=\lambda_{2}, \alpha_{3}^{\prime}=\alpha_{4}^{\prime}=\alpha_{5}^{\prime}=0 .
$$

$\alpha^{\prime}=0$ implies $m_{1}(\alpha)=m_{6}(\alpha)=0$.

We list $\Delta_{k}^{+}$as follows,

$$
\begin{aligned}
& \Delta_{1}^{+}=\left\{\alpha_{2}, \quad \alpha_{4}+\alpha_{2}, \quad \alpha_{3}+\alpha_{4}+\alpha_{2}, \quad \alpha_{4}+\alpha_{5}+\alpha_{2},\right. \\
& \left.\alpha_{3}+\alpha_{4}+\alpha_{5}+\alpha_{2}, \quad \alpha_{3}+2 \alpha_{4}+\alpha_{5}+\alpha_{2}\right\}
\end{aligned}
$$




$$
\begin{aligned}
& \Delta_{2}^{+}=\left\{\alpha_{1}, \quad \alpha_{6}, \quad \alpha_{1}+\alpha_{3}, \quad \alpha_{5}+\alpha_{6},\right. \\
& \alpha_{1}+\alpha_{3}+\alpha_{4}, \quad \alpha_{4}+\alpha_{5}+\alpha_{6}, \\
& \alpha_{1}+\alpha_{3}+\alpha_{4}+\alpha_{5}, \quad \alpha_{3}+\alpha_{4}+\alpha_{5}+\alpha_{6}, \\
&\left.\alpha_{1}+\alpha_{3}+\alpha_{4}+\alpha_{5}+\alpha_{6}\right\} .
\end{aligned}
$$

We get $s_{k}$ as follows,

$$
s_{1}=8, s_{2}=11
$$

Then $s=s_{2}=11$.

$(12) \operatorname{EIV}, \mathfrak{g}^{\mathbb{C}}=\mathfrak{e}_{l}, l=6, r=2$.

we have

$$
\alpha_{1}^{\prime}=\lambda_{1}, \alpha_{6}^{\prime}=\lambda_{2}, \alpha_{2}^{\prime}=\alpha_{3}^{\prime}=\alpha_{4}^{\prime}=\alpha_{5}^{\prime}=0 .
$$

$\alpha^{\prime}=0$ implies $m_{1}(\alpha)=m_{6}(\alpha)=0$.

We list $\Delta_{k}^{+}$as follows,

$$
\begin{aligned}
& \Delta_{1}^{+}=\left\{\alpha_{6}, \quad \alpha_{5}+\alpha_{6}, \quad \alpha_{4}+\alpha_{5}+\alpha_{6},\right. \\
& \alpha_{3}+\alpha_{4}+\alpha_{5}+\alpha_{6}, \quad \alpha_{4}+\alpha_{5}+\alpha_{6}+\alpha_{2}, \\
& \alpha_{3}+\alpha_{4}+\alpha_{5}+\alpha_{6}+\alpha_{2}, \quad \alpha_{3}+2 \alpha_{4}+\alpha_{5}+\alpha_{6}+\alpha_{2}, \\
& \left.\alpha_{3}+2 \alpha_{4}+2 \alpha_{5}+\alpha_{6}+\alpha_{2}\right\} \\
& \Delta_{2}^{+}=\left\{\alpha_{1}, \quad \alpha_{1}+\alpha_{3}, \quad \alpha_{1}+\alpha_{3}+\alpha_{4},\right. \\
& \alpha_{1}+\alpha_{3}+\alpha_{4}+\alpha_{2}, \quad \alpha_{1}+\alpha_{3}+\alpha_{4}+\alpha_{5}, \\
& \alpha_{1}+\alpha_{3}+\alpha_{4}+\alpha_{5}+\alpha_{2}, \quad \alpha_{1}+\alpha_{3}+2 \alpha_{4}+\alpha_{5}+\alpha_{2}, \\
& \left.\alpha_{1}+2 \alpha_{3}+2 \alpha_{4}+\alpha_{5}+\alpha_{2}\right\} \text {. }
\end{aligned}
$$

We get $s_{k}$ as follows,

$$
s_{1}=10, s_{2}=10 .
$$

Then $s=10$.

We draw the Dynkin diagram of $\mathfrak{e}_{7}$ as follows.

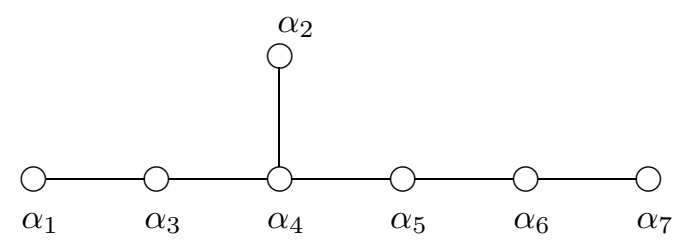

$\mathfrak{e}_{7}$.

$(13) \mathrm{EV}, \mathfrak{g}^{\mathbb{C}}=\mathfrak{e}_{l}, l=7, r=7$.

we have

$$
\alpha_{i}^{\prime}=\lambda_{i}, 1 \leq i \leq 7
$$

$\alpha^{\prime}=0$ implies $\alpha=0$. 
We list $\Delta_{k}^{+}$as follows,

$\Delta_{1}^{+}=\left\{\alpha_{3}, \quad \alpha_{4}, \quad \alpha_{5}, \quad \alpha_{6}, \quad \alpha_{7}, \quad \alpha_{2}\right.$,

$\alpha_{3}+\alpha_{4}, \quad \alpha_{4}+\alpha_{5}, \quad \alpha_{4}+\alpha_{2}, \quad \alpha_{5}+\alpha_{6}, \quad \alpha_{6}+\alpha_{7}$,

$\alpha_{3}+\alpha_{4}+\alpha_{2}, \quad \alpha_{3}+\alpha_{4}+\alpha_{5}, \quad \alpha_{4}+\alpha_{5}+\alpha_{2}, \quad \alpha_{4}+\alpha_{5}+\alpha_{6}, \quad \alpha_{5}+\alpha_{6}+\alpha_{7}$,

$\alpha_{3}+\alpha_{4}+\alpha_{5}+\alpha_{2}, \quad \alpha_{3}+\alpha_{4}+\alpha_{5}+\alpha_{6}, \quad \alpha_{4}+\alpha_{5}+\alpha_{6}+\alpha_{2}$,

$\alpha_{4}+\alpha_{5}+\alpha_{6}+\alpha_{7}, \quad \alpha_{3}+2 \alpha_{4}+\alpha_{5}+\alpha_{2}$,

$\alpha_{3}+\alpha_{4}+\alpha_{5}+\alpha_{6}+\alpha_{2}, \quad \alpha_{3}+\alpha_{4}+\alpha_{5}+\alpha_{6}+\alpha_{7}, \quad \alpha_{4}+\alpha_{5}+\alpha_{6}+\alpha_{7}+\alpha_{2}$,

$\alpha_{3}+2 \alpha_{4}+\alpha_{5}+\alpha_{6}+\alpha_{2}, \quad \alpha_{3}+\alpha_{4}+\alpha_{5}+\alpha_{6}+\alpha_{7}+\alpha_{2}, \quad \alpha_{3}+2 \alpha_{4}+2 \alpha_{5}+\alpha_{6}+\alpha_{2}$,

$\alpha_{3}+2 \alpha_{4}+\alpha_{5}+\alpha_{6}+\alpha_{7}+\alpha_{2}, \quad \alpha_{3}+2 \alpha_{4}+2 \alpha_{5}+\alpha_{6}+\alpha_{7}+\alpha_{2}$,

$\left.\alpha_{3}+2 \alpha_{4}+2 \alpha_{5}+2 \alpha_{6}+\alpha_{7}+\alpha_{2}\right\}$

$\Delta_{2}^{+}=\left\{\alpha_{1}, \quad \alpha_{3}, \quad \alpha_{4}, \quad \alpha_{5}, \quad \alpha_{6}, \quad \alpha_{7}\right.$,

$\alpha_{1}+\alpha_{3}, \quad \alpha_{3}+\alpha_{4}, \quad \alpha_{4}+\alpha_{5}, \quad \alpha_{5}+\alpha_{6}, \quad \alpha_{6}+\alpha_{7}$,

$\alpha_{1}+\alpha_{3}+\alpha_{4}, \quad \alpha_{3}+\alpha_{4}+\alpha_{5}, \quad \alpha_{4}+\alpha_{5}+\alpha_{6}, \quad \alpha_{5}+\alpha_{6}+\alpha_{7}$,

$\alpha_{1}+\alpha_{3}+\alpha_{4}+\alpha_{5}, \quad \alpha_{3}+\alpha_{4}+\alpha_{5}+\alpha_{6}, \quad \alpha_{4}+\alpha_{5}+\alpha_{6}+\alpha_{7}$,

$\left.\alpha_{1}+\alpha_{3}+\alpha_{4}+\alpha_{5}+\alpha_{6}, \quad \alpha_{3}+\alpha_{4}+\alpha_{5}+\alpha_{6}+\alpha_{7}, \quad \alpha_{1}+\alpha_{3}+\alpha_{4}+\alpha_{5}+\alpha_{6}+\alpha_{7}\right\}$

$\Delta_{3}^{+}=\left\{\alpha_{1}, \quad \alpha_{4}, \quad \alpha_{5}, \quad \alpha_{6}, \quad \alpha_{7}, \quad \alpha_{2}\right.$,

$\alpha_{4}+\alpha_{5}, \quad \alpha_{4}+\alpha_{2}, \quad \alpha_{5}+\alpha_{6}, \quad \alpha_{6}+\alpha_{7}$,

$\alpha_{4}+\alpha_{5}+\alpha_{2}, \quad \alpha_{4}+\alpha_{5}+\alpha_{6}, \quad \alpha_{5}+\alpha_{6}+\alpha_{7}$,

$\left.\alpha_{4}+\alpha_{5}+\alpha_{6}+\alpha_{2}, \quad \alpha_{4}+\alpha_{5}+\alpha_{6}+\alpha_{7}, \quad \alpha_{4}+\alpha_{5}+\alpha_{6}+\alpha_{7}+\alpha_{2}\right\}$

$\Delta_{4}^{+}=\left\{\begin{array}{lllll}\alpha_{1}, & \alpha_{3}, & \alpha_{5}, & \alpha_{6}, & \alpha_{7},\end{array} \alpha_{2}\right.$,

$\left.\alpha_{1}+\alpha_{3}, \quad \alpha_{5}+\alpha_{6}, \quad \alpha_{6}+\alpha_{7}, \quad \alpha_{5}+\alpha_{6}+\alpha_{7}\right\}$

$\Delta_{5}^{+}=\left\{\begin{array}{llllll}\alpha_{1}, & \alpha_{3}, & \alpha_{4}, & \alpha_{6}, & \alpha_{7}, & \alpha_{2},\end{array}\right.$

$\alpha_{1}+\alpha_{3}, \quad \alpha_{3}+\alpha_{4}, \quad \alpha_{4}+\alpha_{2}, \quad \alpha_{6}+\alpha_{7}$,

$\left.\alpha_{1}+\alpha_{3}+\alpha_{4}, \quad \alpha_{3}+\alpha_{4}+\alpha_{2}, \quad \alpha_{1}+\alpha_{3}+\alpha_{4}+\alpha_{2}\right\}$

$\Delta_{6}^{+}=\left\{\begin{array}{llllll}\alpha_{1}, & \alpha_{3}, & \alpha_{4}, & \alpha_{5}, & \alpha_{7}, & \alpha_{2},\end{array}\right.$

$\alpha_{1}+\alpha_{3}, \quad \alpha_{3}+\alpha_{4}, \quad \alpha_{4}+\alpha_{5}, \quad \alpha_{4}+\alpha_{2}$,

$\alpha_{1}+\alpha_{3}+\alpha_{4}, \quad \alpha_{3}+\alpha_{4}+\alpha_{2}, \quad \alpha_{3}+\alpha_{4}+\alpha_{5}, \quad \alpha_{4}+\alpha_{5}+\alpha_{2}$,

$\alpha_{1}+\alpha_{3}+\alpha_{4}+\alpha_{2}, \quad \alpha_{1}+\alpha_{3}+\alpha_{4}+\alpha_{5}, \quad \alpha_{3}+\alpha_{4}+\alpha_{5}+\alpha_{2}, \quad \alpha_{3}+2 \alpha_{4}+\alpha_{5}+\alpha_{2}$,

$\left.\alpha_{1}+\alpha_{3}+\alpha_{4}+\alpha_{5}+\alpha_{2}, \quad \alpha_{1}+\alpha_{3}+2 \alpha_{4}+\alpha_{5}+\alpha_{2}, \quad \alpha_{1}+2 \alpha_{3}+2 \alpha_{4}+\alpha_{5}+\alpha_{2}\right\}$

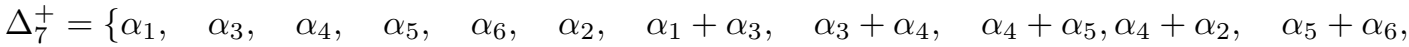

$\alpha_{1}+\alpha_{3}+\alpha_{4}, \quad \alpha_{3}+\alpha_{4}+\alpha_{2}, \quad \alpha_{3}+\alpha_{4}+\alpha_{5}, \quad \alpha_{4}+\alpha_{5}+\alpha_{2}, \quad \alpha_{4}+\alpha_{5}+\alpha_{6}$,

$\alpha_{1}+\alpha_{3}+\alpha_{4}+\alpha_{2}, \quad \alpha_{1}+\alpha_{3}+\alpha_{4}+\alpha_{5}, \quad \alpha_{3}+\alpha_{4}+\alpha_{5}+\alpha_{2}$,

$\alpha_{3}+\alpha_{4}+\alpha_{5}+\alpha_{6}, \quad \alpha_{4}+\alpha_{5}+\alpha_{6}+\alpha_{2}, \quad \alpha_{3}+2 \alpha_{4}+\alpha_{5}+\alpha_{2}$,

$\alpha_{1}+\alpha_{3}+\alpha_{4}+\alpha_{5}+\alpha_{2}, \quad \alpha_{1}+\alpha_{3}+\alpha_{4}+\alpha_{5}+\alpha_{6}, \quad \alpha_{3}+\alpha_{4}+\alpha_{5}+\alpha_{6}+\alpha_{2}$,

$\alpha_{1}+\alpha_{3}+2 \alpha_{4}+\alpha_{5}+\alpha_{2}, \quad \alpha_{1}+\alpha_{3}+\alpha_{4}+\alpha_{5}+\alpha_{6}+\alpha_{2}, \quad \alpha_{3}+2 \alpha_{4}+\alpha_{5}+\alpha_{6}+\alpha_{2}$,

$\alpha_{1}+2 \alpha_{3}+2 \alpha_{4}+\alpha_{5}+\alpha_{2}, \quad \alpha_{1}+\alpha_{3}+2 \alpha_{4}+\alpha_{5}+\alpha_{6}+\alpha_{2}, \quad \alpha_{3}+2 \alpha_{4}+2 \alpha_{5}+\alpha_{6}+\alpha_{2}$,

$\alpha_{1}+2 \alpha_{3}+2 \alpha_{4}+\alpha_{5}+\alpha_{6}+\alpha_{2}, \quad \alpha_{1}+\alpha_{3}+2 \alpha_{4}+2 \alpha_{5}+\alpha_{6}+\alpha_{2}$,

$\alpha_{1}+2 \alpha_{3}+2 \alpha_{4}+2 \alpha_{5}+\alpha_{6}+\alpha_{2}, \quad \alpha_{1}+2 \alpha_{3}+3 \alpha_{4}+2 \alpha_{5}+\alpha_{6}+\alpha_{2}$,

$\left.\alpha_{1}+2 \alpha_{3}+3 \alpha_{4}+2 \alpha_{5}+\alpha_{6}+2 \alpha_{2}\right\}$. 
We get $s_{k}$ as follows,

$$
s_{1}=37, s_{2}=28, s_{3}=23, s_{4}=17, s_{5}=20, s_{6}=28, s_{7}=43 .
$$

Then $s=43$.

(14)EVI, $\mathfrak{g}^{\mathbb{C}}=\mathfrak{e}_{l}, l=7, r=4$.

we have

$$
\alpha_{1}^{\prime}=\lambda_{1}, \alpha_{3}^{\prime}=\lambda_{2}, \alpha_{4}^{\prime}=\lambda_{3}, \alpha_{6}^{\prime}=\lambda_{4}, \alpha_{2}^{\prime}=\alpha_{5}^{\prime}=\alpha_{7}^{\prime}=0 .
$$

$\alpha^{\prime}=0$ implies $m_{2}(\alpha)=m_{5}(\alpha)=m_{7}(\alpha)=0$.

We list $\Delta_{k}^{+}$as follows,

$$
\begin{aligned}
& \Delta_{1}^{+}=\left\{\alpha_{3}, \quad \alpha_{4}, \quad \alpha_{6}, \quad \alpha_{3}+\alpha_{4}, \quad \alpha_{4}+\alpha_{5}, \quad \alpha_{4}+\alpha_{2}, \quad \alpha_{5}+\alpha_{6}, \quad \alpha_{6}+\alpha_{7},\right. \\
& \alpha_{3}+\alpha_{4}+\alpha_{2}, \quad \alpha_{3}+\alpha_{4}+\alpha_{5}, \quad \alpha_{4}+\alpha_{5}+\alpha_{2}, \quad \alpha_{4}+\alpha_{5}+\alpha_{6}, \quad \alpha_{5}+\alpha_{6}+\alpha_{7}, \\
& \alpha_{3}+\alpha_{4}+\alpha_{5}+\alpha_{2}, \quad \alpha_{3}+\alpha_{4}+\alpha_{5}+\alpha_{6}, \quad \alpha_{4}+\alpha_{5}+\alpha_{6}+\alpha_{2}, \quad \alpha_{4}+\alpha_{5}+\alpha_{6}+\alpha_{7}, \\
& \alpha_{3}+2 \alpha_{4}+\alpha_{5}+\alpha_{2}, \quad \alpha_{3}+\alpha_{4}+\alpha_{5}+\alpha_{6}+\alpha_{2}, \quad \alpha_{3}+\alpha_{4}+\alpha_{5}+\alpha_{6}+\alpha_{7}, \\
& \alpha_{4}+\alpha_{5}+\alpha_{6}+\alpha_{7}+\alpha_{2}, \quad \alpha_{3}+2 \alpha_{4}+\alpha_{5}+\alpha_{6}+\alpha_{2}, \quad \alpha_{3}+\alpha_{4}+\alpha_{5}+\alpha_{6}+\alpha_{7}+\alpha_{2}, \\
& \alpha_{3}+2 \alpha_{4}+2 \alpha_{5}+\alpha_{6}+\alpha_{2}, \quad \alpha_{3}+2 \alpha_{4}+\alpha_{5}+\alpha_{6}+\alpha_{7}+\alpha_{2}, \\
& \left.\alpha_{3}+2 \alpha_{4}+2 \alpha_{5}+\alpha_{6}+\alpha_{7}+\alpha_{2}, \quad \alpha_{3}+2 \alpha_{4}+2 \alpha_{5}+2 \alpha_{6}+\alpha_{7}+\alpha_{2}\right\}
\end{aligned}
$$

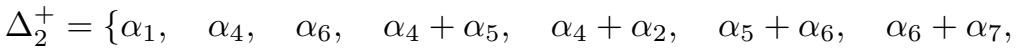

$$
\begin{aligned}
& \alpha_{4}+\alpha_{5}+\alpha_{2}, \quad \alpha_{4}+\alpha_{5}+\alpha_{6}, \quad \alpha_{5}+\alpha_{6}+\alpha_{7}, \\
& \left.\alpha_{4}+\alpha_{5}+\alpha_{6}+\alpha_{2}, \quad \alpha_{4}+\alpha_{5}+\alpha_{6}+\alpha_{7}, \quad \alpha_{4}+\alpha_{5}+\alpha_{6}+\alpha_{7}+\alpha_{2}\right\} \\
& \left.\Delta_{3}^{+}=\alpha_{1}, \quad \alpha_{3}, \quad \alpha_{6}, \quad \alpha_{1}+\alpha_{3}, \quad \alpha_{5}+\alpha_{6}, \quad \alpha_{6}+\alpha_{7}, \quad \alpha_{5}+\alpha_{6}+\alpha_{7}\right\} \\
& \Delta_{4}^{+}=\left\{\alpha_{1}, \quad \alpha_{3}, \quad \alpha_{4}, \quad \alpha_{1}+\alpha_{3}, \quad \alpha_{3}+\alpha_{4}, \quad \alpha_{4}+\alpha_{5}, \quad \alpha_{4}+\alpha_{2},\right. \\
& \alpha_{1}+\alpha_{3}+\alpha_{4}, \quad \alpha_{3}+\alpha_{4}+\alpha_{2}, \quad \alpha_{3}+\alpha_{4}+\alpha_{5}, \quad \alpha_{4}+\alpha_{5}+\alpha_{2}, \\
& \alpha_{1}+\alpha_{3}+\alpha_{4}+\alpha_{2}, \quad \alpha_{1}+\alpha_{3}+\alpha_{4}+\alpha_{5}, \quad \alpha_{3}+\alpha_{4}+\alpha_{5}+\alpha_{2}, \quad \alpha_{3}+2 \alpha_{4}+\alpha_{5}+\alpha_{2}, \\
& \left.\alpha_{1}+\alpha_{3}+\alpha_{4}+\alpha_{5}+\alpha_{2}, \quad \alpha_{1}+\alpha_{3}+2 \alpha_{4}+\alpha_{5}+\alpha_{2}, \quad \alpha_{1}+2 \alpha_{3}+2 \alpha_{4}+\alpha_{5}+\alpha_{2}\right\} .
\end{aligned}
$$

We get $s_{k}$ as follows,

$$
s_{1}=31, s_{2}=17, s_{3}=11, s_{4}=22 .
$$

Then $s=31$.

(15)EVII, $\mathfrak{g}^{\mathbb{C}}=\mathfrak{e}_{l}, l=7, r=3$.

we have

$$
\alpha_{1}^{\prime}=\lambda_{1}, \alpha_{6}^{\prime}=\lambda_{2}, \alpha_{7}^{\prime}=\lambda_{3}, \alpha_{2}^{\prime}=\alpha_{3}^{\prime}=\alpha_{4}^{\prime}=\alpha_{5}^{\prime}=0 .
$$

$\alpha^{\prime}=0$ implies $m_{2}(\alpha)=m_{3}(\alpha)=m_{4}(\alpha)=m_{5}(\alpha)=0$.

We list $\Delta_{k}^{+}$as follows,

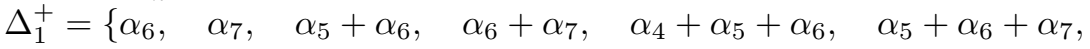

$$
\begin{aligned}
& \alpha_{3}+\alpha_{4}+\alpha_{5}+\alpha_{6}, \quad \alpha_{4}+\alpha_{5}+\alpha_{6}+\alpha_{2}, \quad \alpha_{4}+\alpha_{5}+\alpha_{6}+\alpha_{7}, \\
& \alpha_{3}+\alpha_{4}+\alpha_{5}+\alpha_{6}+\alpha_{2}, \quad \alpha_{3}+\alpha_{4}+\alpha_{5}+\alpha_{6}+\alpha_{7}, \quad \alpha_{4}+\alpha_{5}+\alpha_{6}+\alpha_{7}+\alpha_{2}, \\
& \alpha_{3}+2 \alpha_{4}+\alpha_{5}+\alpha_{6}+\alpha_{2}, \quad \alpha_{3}+\alpha_{4}+\alpha_{5}+\alpha_{6}+\alpha_{7}+\alpha_{2}, \quad \alpha_{3}+2 \alpha_{4}+2 \alpha_{5}+\alpha_{6}+\alpha_{2}, \\
& \alpha_{3}+2 \alpha_{4}+\alpha_{5}+\alpha_{6}+\alpha_{7}+\alpha_{2}, \quad \alpha_{3}+2 \alpha_{4}+2 \alpha_{5}+\alpha_{6}+\alpha_{7}+\alpha_{2}, \\
& \left.\alpha_{3}+2 \alpha_{4}+2 \alpha_{5}+2 \alpha_{6}+\alpha_{7}+\alpha_{2}\right\} \\
& \Delta_{2}^{+}=\left\{\alpha_{1}, \quad \alpha_{7}, \quad \alpha_{1}+\alpha_{3}, \quad \alpha_{1}+\alpha_{3}+\alpha_{4},\right. \\
& \alpha_{1}+\alpha_{3}+\alpha_{4}+\alpha_{2}, \quad \alpha_{1}+\alpha_{3}+\alpha_{4}+\alpha_{5}, \\
& \left.\alpha_{1}+\alpha_{3}+\alpha_{4}+\alpha_{5}+\alpha_{2}, \quad \alpha_{1}+\alpha_{3}+2 \alpha_{4}+\alpha_{5}+\alpha_{2}, \quad \alpha_{1}+2 \alpha_{3}+2 \alpha_{4}+\alpha_{5}+\alpha_{2}\right\}
\end{aligned}
$$




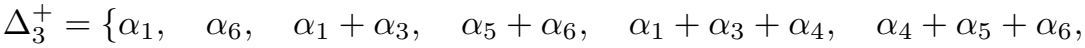

$$
\begin{aligned}
& \alpha_{1}+\alpha_{3}+\alpha_{4}+\alpha_{2}, \quad \alpha_{1}+\alpha_{3}+\alpha_{4}+\alpha_{5}, \quad \alpha_{3}+\alpha_{4}+\alpha_{5}+\alpha_{6}, \\
& \alpha_{4}+\alpha_{5}+\alpha_{6}+\alpha_{2}, \quad \alpha_{1}+\alpha_{3}+\alpha_{4}+\alpha_{5}+\alpha_{2}, \quad \alpha_{1}+\alpha_{3}+\alpha_{4}+\alpha_{5}+\alpha_{6}, \\
& \alpha_{3}+\alpha_{4}+\alpha_{5}+\alpha_{6}+\alpha_{2}, \quad \alpha_{1}+\alpha_{3}+2 \alpha_{4}+\alpha_{5}+\alpha_{2}, \quad \alpha_{1}+\alpha_{3}+\alpha_{4}+\alpha_{5}+\alpha_{6}+\alpha_{2}, \\
& \alpha_{3}+2 \alpha_{4}+\alpha_{5}+\alpha_{6}+\alpha_{2}, \quad \alpha_{1}+2 \alpha_{3}+2 \alpha_{4}+\alpha_{5}+\alpha_{2}, \quad \alpha_{1}+\alpha_{3}+2 \alpha_{4}+\alpha_{5}+\alpha_{6}+\alpha_{2}, \\
& \alpha_{3}+2 \alpha_{4}+2 \alpha_{5}+\alpha_{6}+\alpha_{2}, \quad \alpha_{1}+2 \alpha_{3}+2 \alpha_{4}+\alpha_{5}+\alpha_{6}+\alpha_{2}, \quad \alpha_{1}+\alpha_{3}+2 \alpha_{4}+2 \alpha_{5}+\alpha_{6}+\alpha_{2} \text {, } \\
& \alpha_{1}+2 \alpha_{3}+2 \alpha_{4}+2 \alpha_{5}+\alpha_{6}+\alpha_{2}, \quad \alpha_{1}+2 \alpha_{3}+3 \alpha_{4}+2 \alpha_{5}+\alpha_{6}+\alpha_{2} \text {, } \\
& \left.\alpha_{1}+2 \alpha_{3}+3 \alpha_{4}+2 \alpha_{5}+\alpha_{6}+2 \alpha_{2}\right\} \text {. }
\end{aligned}
$$

We get $s_{k}$ as follows,

$$
s_{1}=21, s_{2}=12, s_{3}=27 \text {. }
$$

Then $s=27$.

We draw the Dynkin diagram of $\mathfrak{e}_{8}$ as follows.

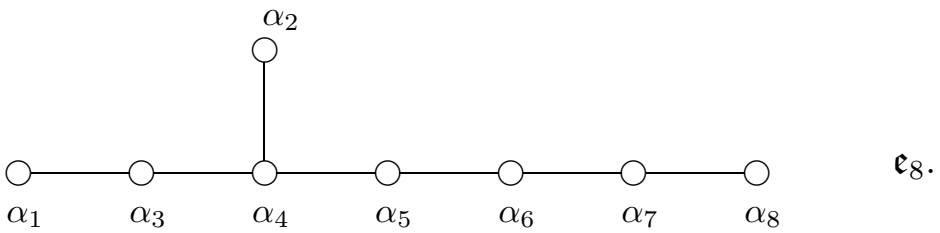

(16) EVIII, $\mathfrak{g}^{\mathbb{C}}=\mathfrak{e}_{8}, l=8, r=8$.

we have

$$
\alpha_{i}^{\prime}=\lambda_{i}, 1 \leq i \leq 8
$$

$\alpha^{\prime}=0$ implies $\alpha=0$.

We list $\Delta_{k}^{+}$as follows,

$$
\begin{aligned}
& \Delta_{1}^{+}=\left\{\alpha_{3}, \quad \alpha_{4}, \quad \alpha_{5}, \quad \alpha_{6}, \quad \alpha_{7}, \quad \alpha_{8}, \quad \alpha_{2},\right. \\
& \alpha_{3}+\alpha_{4}, \quad \alpha_{4}+\alpha_{2}, \quad \alpha_{4}+\alpha_{5}, \quad \alpha_{5}+\alpha_{6}, \quad \alpha_{6}+\alpha_{7}, \quad \alpha_{7}+\alpha_{8}, \\
& \alpha_{3}+\alpha_{4}+\alpha_{2}, \quad \alpha_{3}+\alpha_{4}+\alpha_{5}, \quad \alpha_{4}+\alpha_{5}+\alpha_{2}, \\
& \alpha_{4}+\alpha_{5}+\alpha_{6}, \quad \alpha_{5}+\alpha_{6}+\alpha_{7}, \quad \alpha_{6}+\alpha_{7}+\alpha_{8} \\
& \alpha_{3}+\alpha_{4}+\alpha_{5}+\alpha_{2}, \quad \alpha_{3}+\alpha_{4}+\alpha_{5}+\alpha_{6}, \quad \alpha_{4}+\alpha_{5}+\alpha_{6}+\alpha_{2}, \\
& \alpha_{4}+\alpha_{5}+\alpha_{6}+\alpha_{7}, \quad \alpha_{5}+\alpha_{6}+\alpha_{7}+\alpha_{8}, \quad \alpha_{3}+2 \alpha_{4}+\alpha_{5}+\alpha_{2}, \\
& \alpha_{3}+\alpha_{4}+\alpha_{5}+\alpha_{6}+\alpha_{2}, \quad \alpha_{3}+\alpha_{4}+\alpha_{5}+\alpha_{6}+\alpha_{7}, \quad \alpha_{4}+\alpha_{5}+\alpha_{6}+\alpha_{7}+\alpha_{2}, \\
& \alpha_{4}+\alpha_{5}+\alpha_{6}+\alpha_{7}+\alpha_{8}, \quad \alpha_{3}+2 \alpha_{4}+\alpha_{5}+\alpha_{6}+\alpha_{2}, \quad \alpha_{3}+\alpha_{4}+\alpha_{5}+\alpha_{6}+\alpha_{7}+\alpha_{2}, \\
& \alpha_{3}+\alpha_{4}+\alpha_{5}+\alpha_{6}+\alpha_{7}+\alpha_{8}, \quad \alpha_{4}+\alpha_{5}+\alpha_{6}+\alpha_{7}+\alpha_{8}+\alpha_{2}, \\
& \alpha_{3}+2 \alpha_{4}+2 \alpha_{5}+\alpha_{6}+\alpha_{2}, \alpha_{3}+2 \alpha_{4}+\alpha_{5}+\alpha_{6}+\alpha_{7}+\alpha_{2} \text {, } \\
& \alpha_{3}+\alpha_{4}+\alpha_{5}+\alpha_{6}+\alpha_{7}+\alpha_{8}+\alpha_{2}, \quad \alpha_{3}+2 \alpha_{4}+2 \alpha_{5}+\alpha_{6}+\alpha_{7}+\alpha_{2}, \\
& \alpha_{3}+2 \alpha_{4}+\alpha_{5}+\alpha_{6}+\alpha_{7}+\alpha_{8}+\alpha_{2}, \quad \alpha_{3}+2 \alpha_{4}+2 \alpha_{5}+2 \alpha_{6}+\alpha_{7}+\alpha_{2}, \\
& \alpha_{3}+2 \alpha_{4}+2 \alpha_{5}+\alpha_{6}+\alpha_{7}+\alpha_{8}+\alpha_{2}, \quad \alpha_{3}+2 \alpha_{4}+2 \alpha_{5}+2 \alpha_{6}+\alpha_{7}+\alpha_{8}+\alpha_{2}, \\
& \left.\alpha_{3}+2 \alpha_{4}+2 \alpha_{5}+2 \alpha_{6}+2 \alpha_{7}+\alpha_{8}+\alpha_{2}\right\}
\end{aligned}
$$




$$
\begin{aligned}
& \Delta_{2}^{+}=\left\{\alpha_{1}, \quad \alpha_{3}, \quad \alpha_{4}, \quad \alpha_{5}, \quad \alpha_{6}, \quad \alpha_{7}, \quad \alpha_{8},\right. \\
& \alpha_{1}+\alpha_{3}, \quad \alpha_{3}+\alpha_{4}, \quad \alpha_{4}+\alpha_{5}, \quad \alpha_{5}+\alpha_{6}, \quad \alpha_{6}+\alpha_{7}, \quad \alpha_{7}+\alpha_{8}, \\
& \alpha_{1}+\alpha_{3}+\alpha_{4}, \quad \alpha_{3}+\alpha_{4}+\alpha_{5}, \quad \alpha_{4}+\alpha_{5}+\alpha_{6}, \\
& \alpha_{5}+\alpha_{6}+\alpha_{7}, \quad \alpha_{6}+\alpha_{7}+\alpha_{8}, \quad \alpha_{1}+\alpha_{3}+\alpha_{4}+\alpha_{5}, \\
& \alpha_{3}+\alpha_{4}+\alpha_{5}+\alpha_{6}, \quad \alpha_{4}+\alpha_{5}+\alpha_{6}+\alpha_{7}, \quad \alpha_{5}+\alpha_{6}+\alpha_{7}+\alpha_{8}, \\
& \alpha_{1}+\alpha_{3}+\alpha_{4}+\alpha_{5}+\alpha_{6}, \quad \alpha_{3}+\alpha_{4}+\alpha_{5}+\alpha_{6}+\alpha_{7}, \quad \alpha_{4}+\alpha_{5}+\alpha_{6}+\alpha_{7}+\alpha_{8}, \\
& \alpha_{1}+\alpha_{3}+\alpha_{4}+\alpha_{5}+\alpha_{6}+\alpha_{7}, \quad \alpha_{3}+\alpha_{4}+\alpha_{5}+\alpha_{6}+\alpha_{7}+\alpha_{8}, \\
& \left.\alpha_{1}+\alpha_{3}+\alpha_{4}+\alpha_{5}+\alpha_{6}+\alpha_{7}+\alpha_{8}\right\}
\end{aligned}
$$

$\Delta_{3}^{+}=\left\{\alpha_{1}, \quad \alpha_{4}, \quad \alpha_{5}, \quad \alpha_{6}, \quad \alpha_{7}, \quad \alpha_{8}, \quad \alpha_{2}\right.$,

$\alpha_{4}+\alpha_{2}, \quad \alpha_{4}+\alpha_{5}, \quad \alpha_{5}+\alpha_{6}, \quad \alpha_{6}+\alpha_{7}, \quad \alpha_{7}+\alpha_{8}$,

$\alpha_{4}+\alpha_{5}+\alpha_{2}, \quad \alpha_{4}+\alpha_{5}+\alpha_{6}, \quad \alpha_{5}+\alpha_{6}+\alpha_{7}, \quad \alpha_{6}+\alpha_{7}+\alpha_{8}$,

$\alpha_{4}+\alpha_{5}+\alpha_{6}+\alpha_{2}, \quad \alpha_{4}+\alpha_{5}+\alpha_{6}+\alpha_{7}, \quad \alpha_{5}+\alpha_{6}+\alpha_{7}+\alpha_{8}$,

$\left.\alpha_{4}+\alpha_{5}+\alpha_{6}+\alpha_{7}+\alpha_{2}, \quad \alpha_{4}+\alpha_{5}+\alpha_{6}+\alpha_{7}+\alpha_{8}, \quad \alpha_{4}+\alpha_{5}+\alpha_{6}+\alpha_{7}+\alpha_{8}+\alpha_{2}\right\}$

$\Delta_{4}^{+}=\left\{\begin{array}{lllll}\alpha_{1}, & \alpha_{3}, & \alpha_{5}, & \alpha_{6}, & \alpha_{7},\end{array} \alpha_{8}, \quad \alpha_{2}\right.$,

quad $\alpha_{1}+\alpha_{3}, \quad \alpha_{5}+\alpha_{6}, \quad \alpha_{6}+\alpha_{7}, \quad \alpha_{7}+\alpha_{8}$,

$\left.\alpha_{5}+\alpha_{6}+\alpha_{7}, \quad \alpha_{6}+\alpha_{7}+\alpha_{8}, \quad \alpha_{5}+\alpha_{6}+\alpha_{7}+\alpha_{8}\right\}$

$\Delta_{5}^{+}=\left\{\begin{array}{lllll}\alpha_{1}, & \alpha_{3}, & \alpha_{4}, & \alpha_{6}, & \alpha_{7},\end{array} \alpha_{8}, \quad \alpha_{2}\right.$,

$\alpha_{1}+\alpha_{3}, \quad \alpha_{3}+\alpha_{4}, \quad \alpha_{4}+\alpha_{2}, \quad \alpha_{6}+\alpha_{7}, \quad \alpha_{7}+\alpha_{8}$,

$\alpha_{1}+\alpha_{3}+\alpha_{4}, \quad \alpha_{3}+\alpha_{4}+\alpha_{2}, \quad \alpha_{6}+\alpha_{7}+\alpha_{8}$,

$\left.\alpha_{1}+\alpha_{3}+\alpha_{4}+\alpha_{2}\right\}$

$\Delta_{6}^{+}=\left\{\begin{array}{lllll}\alpha_{1}, & \alpha_{3}, & \alpha_{4}, & \alpha_{5}, & \alpha_{7},\end{array} \alpha_{8}, \quad \alpha_{2}\right.$,

$\alpha_{1}+\alpha_{3}, \quad \alpha_{3}+\alpha_{4}, \quad \alpha_{4}+\alpha_{2}, \quad \alpha_{4}+\alpha_{5}, \quad \alpha_{7}+\alpha_{8}$,

$\alpha_{1}+\alpha_{3}+\alpha_{4}, \quad \alpha_{3}+\alpha_{4}+\alpha_{2}, \quad \alpha_{3}+\alpha_{4}+\alpha_{5}, \quad \alpha_{4}+\alpha_{5}+\alpha_{2}$,

$\alpha_{1}+\alpha_{3}+\alpha_{4}+\alpha_{5}, \quad \alpha_{1}+\alpha_{3}+\alpha_{4}+\alpha_{2}, \quad \alpha_{3}+\alpha_{4}+\alpha_{5}+\alpha_{2}$,

$\alpha_{1}+\alpha_{3}+\alpha_{4}+\alpha_{5}+\alpha_{2}, \quad \alpha_{3}+2 \alpha_{4}+\alpha_{5}+\alpha_{2}, \quad \alpha_{1}+\alpha_{3}+2 \alpha_{4}+\alpha_{5}+\alpha_{2}$,

$\left.\alpha_{1}+2 \alpha_{3}+2 \alpha_{4}+\alpha_{5}+\alpha_{2}\right\}$

$\Delta_{7}^{+}=\left\{\begin{array}{lllll}\alpha_{1}, & \alpha_{3}, & \alpha_{4}, & \alpha_{5}, & \alpha_{6},\end{array} \alpha_{8}, \quad \alpha_{2}\right.$,

$\alpha_{1}+\alpha_{3}, \quad \alpha_{3}+\alpha_{4}, \quad \alpha_{4}+\alpha_{2}, \quad \alpha_{4}+\alpha_{5}, \quad \alpha_{5}+\alpha_{6}$,

$\alpha_{1}+\alpha_{3}+\alpha_{4}, \quad \alpha_{3}+\alpha_{4}+\alpha_{2}, \quad \alpha_{3}+\alpha_{4}+\alpha_{5}$,

$\alpha_{4}+\alpha_{5}+\alpha_{2}, \quad \alpha_{4}+\alpha_{5}+\alpha_{6}$,

$\alpha_{1}+\alpha_{3}+\alpha_{4}+\alpha_{5}, \quad \alpha_{1}+\alpha_{3}+\alpha_{4}+\alpha_{2}, \quad \alpha_{3}+\alpha_{4}+\alpha_{5}+\alpha_{2}$,

$\alpha_{3}+\alpha_{4}+\alpha_{5}+\alpha_{6}, \quad \alpha_{4}+\alpha_{5}+\alpha_{6}+\alpha_{2}$,

$\alpha_{1}+\alpha_{3}+\alpha_{4}+\alpha_{5}+\alpha_{2}, \quad \alpha_{3}+2 \alpha_{4}+\alpha_{5}+\alpha_{2}, \quad \alpha_{1}+\alpha_{3}+\alpha_{4}+\alpha_{5}+\alpha_{6}$,

$\alpha_{3}+\alpha_{4}+\alpha_{5}+\alpha_{6}+\alpha_{2}, \quad \alpha_{1}+\alpha_{3}+2 \alpha_{4}+\alpha_{5}+\alpha_{2}, \quad \alpha_{1}+\alpha_{3}+\alpha_{4}+\alpha_{5}+\alpha_{6}+\alpha_{2}$,

$\alpha_{3}+2 \alpha_{4}+\alpha_{5}+\alpha_{6}+\alpha_{2}, \quad \alpha_{1}+2 \alpha_{3}+2 \alpha_{4}+\alpha_{5}+\alpha_{2}, \quad \alpha_{1}+\alpha_{3}+2 \alpha_{4}+\alpha_{5}+\alpha_{6}+\alpha_{2}$,

$\alpha_{3}+2 \alpha_{4}+2 \alpha_{5}+\alpha_{6}+\alpha_{2}, \quad \alpha_{1}+2 \alpha_{3}+2 \alpha_{4}+\alpha_{5}+\alpha_{6}+\alpha_{2}$,

$\alpha_{1}+\alpha_{3}+2 \alpha_{4}+2 \alpha_{5}+\alpha_{6}+\alpha_{2}, \alpha_{1}+2 \alpha_{3}+2 \alpha_{4}+2 \alpha_{5}+\alpha_{6}+\alpha_{2}$,

$\left.\alpha_{1}+2 \alpha_{3}+3 \alpha_{4}+2 \alpha_{5}+\alpha_{6}+\alpha_{2}, \quad \alpha_{1}+2 \alpha_{3}+3 \alpha_{4}+2 \alpha_{5}+\alpha_{6}+2 \alpha_{2}\right\}$ 


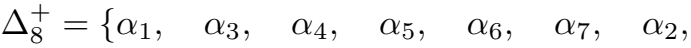

$$
\begin{aligned}
& \alpha_{1}+\alpha_{3}, \quad \alpha_{3}+\alpha_{4}, \quad \alpha_{4}+\alpha_{2}, \quad \alpha_{4}+\alpha_{5}, \quad \alpha_{5}+\alpha_{6}, \alpha_{6}+\alpha_{7}, \\
& \alpha_{1}+\alpha_{3}+\alpha_{4}, \quad \alpha_{3}+\alpha_{4}+\alpha_{2}, \quad \alpha_{3}+\alpha_{4}+\alpha_{5}, \\
& \alpha_{4}+\alpha_{5}+\alpha_{2}, \quad \alpha_{4}+\alpha_{5}+\alpha_{6}, \quad \alpha_{5}+\alpha_{6}+\alpha_{7}, \\
& \alpha_{1}+\alpha_{3}+\alpha_{4}+\alpha_{5}, \quad \alpha_{1}+\alpha_{3}+\alpha_{4}+\alpha_{2}, \quad \alpha_{3}+\alpha_{4}+\alpha_{5}+\alpha_{2}, \\
& \alpha_{3}+\alpha_{4}+\alpha_{5}+\alpha_{6}, \quad \alpha_{4}+\alpha_{5}+\alpha_{6}+\alpha_{2}, \quad \alpha_{4}+\alpha_{5}+\alpha_{6}+\alpha_{7}, \\
& \alpha_{1}+\alpha_{3}+\alpha_{4}+\alpha_{5}+\alpha_{2}, \quad \alpha_{3}+2 \alpha_{4}+\alpha_{5}+\alpha_{2}, \quad \alpha_{1}+\alpha_{3}+\alpha_{4}+\alpha_{5}+\alpha_{6}, \\
& \alpha_{3}+\alpha_{4}+\alpha_{5}+\alpha_{6}+\alpha_{2}, \quad \alpha_{3}+\alpha_{4}+\alpha_{5}+\alpha_{6}+\alpha_{7}, \quad \alpha_{4}+\alpha_{5}+\alpha_{6}+\alpha_{7}+\alpha_{2}, \\
& \alpha_{1}+\alpha_{3}+2 \alpha_{4}+\alpha_{5}+\alpha_{2}, \quad \alpha_{1}+\alpha_{3}+\alpha_{4}+\alpha_{5}+\alpha_{6}+\alpha_{2}, \quad \alpha_{3}+2 \alpha_{4}+\alpha_{5}+\alpha_{6}+\alpha_{2}, \\
& \alpha_{1}+\alpha_{3}+\alpha_{4}+\alpha_{5}+\alpha_{6}+\alpha_{7}, \quad \alpha_{3}+\alpha_{4}+\alpha_{5}+\alpha_{6}+\alpha_{7}+\alpha_{2}, \quad \alpha_{1}+2 \alpha_{3}+2 \alpha_{4}+\alpha_{5}+\alpha_{2}, \\
& \alpha_{1}+\alpha_{3}+2 \alpha_{4}+\alpha_{5}+\alpha_{6}+\alpha_{2}, \quad \alpha_{3}+2 \alpha_{4}+2 \alpha_{5}+\alpha_{6}+\alpha_{2}, \\
& \alpha_{1}+\alpha_{3}+\alpha_{4}+\alpha_{5}+\alpha_{6}+\alpha_{7}+\alpha_{2}, \quad \alpha_{3}+2 \alpha_{4}+\alpha_{5}+\alpha_{6}+\alpha_{7}+\alpha_{2}, \\
& \alpha_{1}+2 \alpha_{3}+2 \alpha_{4}+\alpha_{5}+\alpha_{6}+\alpha_{2}, \quad \alpha_{1}+\alpha_{3}+2 \alpha_{4}+2 \alpha_{5}+\alpha_{6}+\alpha_{2}, \\
& \alpha_{1}+\alpha_{3}+2 \alpha_{4}+\alpha_{5}+\alpha_{6}+\alpha_{7}+\alpha_{2}, \quad \alpha_{3}+2 \alpha_{4}+2 \alpha_{5}+\alpha_{6}+\alpha_{7}+\alpha_{2}, \\
& \alpha_{1}+2 \alpha_{3}+2 \alpha_{4}+2 \alpha_{5}+\alpha_{6}+\alpha_{2}, \quad \alpha_{1}+2 \alpha_{3}+2 \alpha_{4}+\alpha_{5}+\alpha_{6}+\alpha_{7}+\alpha_{2}, \\
& \alpha_{1}+\alpha_{3}+2 \alpha_{4}+2 \alpha_{5}+\alpha_{6}+\alpha_{7}+\alpha_{2}, \quad \alpha_{3}+2 \alpha_{4}+2 \alpha_{5}+2 \alpha_{6}+\alpha_{7}+\alpha_{2}, \\
& \alpha_{1}+2 \alpha_{3}+3 \alpha_{4}+2 \alpha_{5}+\alpha_{6}+\alpha_{2}, \quad \alpha_{1}+2 \alpha_{3}+2 \alpha_{4}+2 \alpha_{5}+\alpha_{6}+\alpha_{7}+\alpha_{2}, \\
& \alpha_{1}+\alpha_{3}+2 \alpha_{4}+2 \alpha_{5}+2 \alpha_{6}+\alpha_{7}+\alpha_{2}, \quad \alpha_{1}+2 \alpha_{3}+3 \alpha_{4}+2 \alpha_{5}+\alpha_{6}+2 \alpha_{2} \text {, } \\
& \alpha_{1}+2 \alpha_{3}+3 \alpha_{4}+2 \alpha_{5}+\alpha_{6}+\alpha_{7}+\alpha_{2}, \quad \alpha_{1}+2 \alpha_{3}+2 \alpha_{4}+2 \alpha_{5}+2 \alpha_{6}+\alpha_{7}+\alpha_{2}, \\
& \alpha_{1}+2 \alpha_{3}+3 \alpha_{4}+2 \alpha_{5}+\alpha_{6}+\alpha_{7}+2 \alpha_{2}, \quad \alpha_{1}+2 \alpha_{3}+3 \alpha_{4}+2 \alpha_{5}+2 \alpha_{6}+\alpha_{7}+\alpha_{2}, \\
& \alpha_{1}+2 \alpha_{3}+3 \alpha_{4}+2 \alpha_{5}+2 \alpha_{6}+\alpha_{7}+2 \alpha_{2}, \quad \alpha_{1}+2 \alpha_{3}+3 \alpha_{4}+3 \alpha_{5}+2 \alpha_{6}+\alpha_{7}+\alpha_{2}, \\
& \alpha_{1}+2 \alpha_{3}+3 \alpha_{4}+3 \alpha_{5}+2 \alpha_{6}+\alpha_{7}+2 \alpha_{2}, \quad \alpha_{1}+2 \alpha_{3}+4 \alpha_{4}+3 \alpha_{5}+2 \alpha_{6}+\alpha_{7}+2 \alpha_{2} \text {, } \\
& \left.\alpha_{1}+3 \alpha_{3}+4 \alpha_{4}+3 \alpha_{5}+2 \alpha_{6}+\alpha_{7}+2 \alpha_{2}, \quad 2 \alpha_{1}+3 \alpha_{3}+4 \alpha_{4}+3 \alpha_{5}+2 \alpha_{6}+\alpha_{7}+2 \alpha_{2}\right\}
\end{aligned}
$$

We get $s_{k}$ as follows,

$$
s_{1}=50, s_{2}=36, s_{3}=30, s_{4}=22, s_{5}=24, s_{6}=31, s_{7}=45, s_{8}=71
$$

Then $s=71$.

(17) EIX, $\mathfrak{g}^{\mathbb{C}}=\mathfrak{e}_{8}, l=8, r=4$.

we have

$$
\alpha_{1}^{\prime}=\lambda_{1}, \alpha_{6}^{\prime}=\lambda_{2}, \alpha_{7}^{\prime}=\lambda_{3}, \alpha_{8}^{\prime}=\lambda_{4}, \alpha_{2}^{\prime}=\alpha_{3}^{\prime}=\alpha_{4}^{\prime}=\alpha_{5}^{\prime}=0
$$

$\alpha^{\prime}=0$ implies $m_{2}(\alpha)=m_{3}(\alpha)=m_{4}(\alpha)=m_{5}(\alpha)=0$. 
We list $\Delta_{k}^{+}$as follows,

$$
\begin{aligned}
& \Delta_{1}^{+}=\left\{\alpha_{6}, \quad \alpha_{7}, \quad \alpha_{8}, \quad \alpha_{5}+\alpha_{6}, \quad \alpha_{6}+\alpha_{7}, \quad \alpha_{7}+\alpha_{8},\right. \\
& \alpha_{4}+\alpha_{5}+\alpha_{6}, \quad \alpha_{5}+\alpha_{6}+\alpha_{7}, \quad \alpha_{6}+\alpha_{7}+\alpha_{8}, \\
& \alpha_{3}+\alpha_{4}+\alpha_{5}+\alpha_{6}, \quad \alpha_{4}+\alpha_{5}+\alpha_{6}+\alpha_{2}, \quad \alpha_{4}+\alpha_{5}+\alpha_{6}+\alpha_{7}, \\
& \alpha_{5}+\alpha_{6}+\alpha_{7}+\alpha_{8}, \quad \alpha_{3}+\alpha_{4}+\alpha_{5}+\alpha_{6}+\alpha_{2}, \quad \alpha_{3}+\alpha_{4}+\alpha_{5}+\alpha_{6}+\alpha_{7}, \\
& \alpha_{4}+\alpha_{5}+\alpha_{6}+\alpha_{7}+\alpha_{2}, \quad \alpha_{4}+\alpha_{5}+\alpha_{6}+\alpha_{7}+\alpha_{8}, \quad \alpha_{3}+2 \alpha_{4}+\alpha_{5}+\alpha_{6}+\alpha_{2}, \\
& \alpha_{3}+\alpha_{4}+\alpha_{5}+\alpha_{6}+\alpha_{7}+\alpha_{2}, \quad \alpha_{3}+\alpha_{4}+\alpha_{5}+\alpha_{6}+\alpha_{7}+\alpha_{8}, \quad \alpha_{4}+\alpha_{5}+\alpha_{6}+\alpha_{7}+\alpha_{8}+\alpha_{2}, \\
& \alpha_{3}+2 \alpha_{4}+2 \alpha_{5}+\alpha_{6}+\alpha_{2}, \quad \alpha_{3}+2 \alpha_{4}+\alpha_{5}+\alpha_{6}+\alpha_{7}+\alpha_{2}, \\
& \alpha_{3}+\alpha_{4}+\alpha_{5}+\alpha_{6}+\alpha_{7}+\alpha_{8}+\alpha_{2}, \quad \alpha_{3}+2 \alpha_{4}+2 \alpha_{5}+\alpha_{6}+\alpha_{7}+\alpha_{2}, \\
& \alpha_{3}+2 \alpha_{4}+\alpha_{5}+\alpha_{6}+\alpha_{7}+\alpha_{8}+\alpha_{2}, \quad \alpha_{3}+2 \alpha_{4}+2 \alpha_{5}+2 \alpha_{6}+\alpha_{7}+\alpha_{2}, \\
& \alpha_{3}+2 \alpha_{4}+2 \alpha_{5}+\alpha_{6}+\alpha_{7}+\alpha_{8}+\alpha_{2}, \quad \alpha_{3}+2 \alpha_{4}+2 \alpha_{5}+2 \alpha_{6}+\alpha_{7}+\alpha_{8}+\alpha_{2}, \\
& \left.\alpha_{3}+2 \alpha_{4}+2 \alpha_{5}+2 \alpha_{6}+2 \alpha_{7}+\alpha_{8}+\alpha_{2}\right\}
\end{aligned}
$$

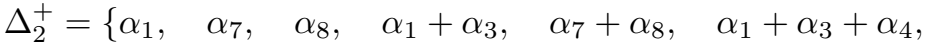

$$
\begin{aligned}
& \alpha_{1}+\alpha_{3}+\alpha_{4}+\alpha_{5}, \quad \alpha_{1}+\alpha_{3}+\alpha_{4}+\alpha_{2}, \quad \alpha_{1}+\alpha_{3}+\alpha_{4}+\alpha_{5}+\alpha_{2}, \\
& \left.\alpha_{1}+\alpha_{3}+2 \alpha_{4}+\alpha_{5}+\alpha_{2}, \quad \alpha_{1}+2 \alpha_{3}+2 \alpha_{4}+\alpha_{5}+\alpha_{2}\right\}
\end{aligned}
$$

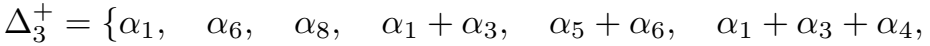

$$
\begin{aligned}
& \alpha_{4}+\alpha_{5}+\alpha_{6}, \quad \alpha_{1}+\alpha_{3}+\alpha_{4}+\alpha_{5}, \quad \alpha_{1}+\alpha_{3}+\alpha_{4}+\alpha_{2}, \\
& \alpha_{3}+\alpha_{4}+\alpha_{5}+\alpha_{6}, \quad \alpha_{4}+\alpha_{5}+\alpha_{6}+\alpha_{2}, \quad \alpha_{1}+\alpha_{3}+\alpha_{4}+\alpha_{5}+\alpha_{2}, \\
& \alpha_{1}+\alpha_{3}+\alpha_{4}+\alpha_{5}+\alpha_{6}, \quad \alpha_{3}+\alpha_{4}+\alpha_{5}+\alpha_{6}+\alpha_{2}, \quad \alpha_{1}+\alpha_{3}+2 \alpha_{4}+\alpha_{5}+\alpha_{2}, \\
& \alpha_{1}+\alpha_{3}+\alpha_{4}+\alpha_{5}+\alpha_{6}+\alpha_{2}, \quad \alpha_{3}+2 \alpha_{4}+\alpha_{5}+\alpha_{6}+\alpha_{2}, \\
& \alpha_{1}+2 \alpha_{3}+2 \alpha_{4}+\alpha_{5}+\alpha_{2}, \quad \alpha_{1}+\alpha_{3}+2 \alpha_{4}+\alpha_{5}+\alpha_{6}+\alpha_{2}, \\
& \alpha_{3}+2 \alpha_{4}+2 \alpha_{5}+\alpha_{6}+\alpha_{2}, \quad \alpha_{1}+2 \alpha_{3}+2 \alpha_{4}+\alpha_{5}+\alpha_{6}+\alpha_{2}, \\
& \alpha_{1}+\alpha_{3}+2 \alpha_{4}+2 \alpha_{5}+\alpha_{6}+\alpha_{2}, \quad \alpha_{1}+2 \alpha_{3}+2 \alpha_{4}+2 \alpha_{5}+\alpha_{6}+\alpha_{2}, \\
& \left.\alpha_{1}+2 \alpha_{3}+3 \alpha_{4}+2 \alpha_{5}+\alpha_{6}+\alpha_{2}, \quad \alpha_{1}+2 \alpha_{3}+3 \alpha_{4}+2 \alpha_{5}+\alpha_{6}+2 \alpha_{2}\right\}
\end{aligned}
$$$$
\Delta_{4}^{+}=\left\{\begin{array}{lllll}
\alpha_{1}, & \alpha_{6}, & \alpha_{7}, & \alpha_{1}+\alpha_{3}, & \alpha_{5}+\alpha_{6},
\end{array} \alpha_{6}+\alpha_{7},\right.
$$$$
\alpha_{1}+\alpha_{3}+\alpha_{4}, \quad \alpha_{4}+\alpha_{5}+\alpha_{6}, \quad \alpha_{5}+\alpha_{6}+\alpha_{7},
$$$$
\alpha_{1}+\alpha_{3}+\alpha_{4}+\alpha_{5}, \quad \alpha_{1}+\alpha_{3}+\alpha_{4}+\alpha_{2}, \quad \alpha_{3}+\alpha_{4}+\alpha_{5}+\alpha_{6},
$$$$
\alpha_{4}+\alpha_{5}+\alpha_{6}+\alpha_{2}, \quad \alpha_{4}+\alpha_{5}+\alpha_{6}+\alpha_{7}, \quad \alpha_{1}+\alpha_{3}+\alpha_{4}+\alpha_{5}+\alpha_{2},
$$$$
\alpha_{1}+\alpha_{3}+\alpha_{4}+\alpha_{5}+\alpha_{6}, \quad \alpha_{3}+\alpha_{4}+\alpha_{5}+\alpha_{6}+\alpha_{2}, \quad \alpha_{3}+\alpha_{4}+\alpha_{5}+\alpha_{6}+\alpha_{7},
$$$$
\alpha_{4}+\alpha_{5}+\alpha_{6}+\alpha_{7}+\alpha_{2}, \quad \alpha_{1}+\alpha_{3}+2 \alpha_{4}+\alpha_{5}+\alpha_{2},
$$$$
\alpha_{1}+\alpha_{3}+\alpha_{4}+\alpha_{5}+\alpha_{6}+\alpha_{2}, \quad \alpha_{3}+2 \alpha_{4}+\alpha_{5}+\alpha_{6}+\alpha_{2},
$$$$
\alpha_{1}+\alpha_{3}+\alpha_{4}+\alpha_{5}+\alpha_{6}+\alpha_{7}, \quad \alpha_{3}+\alpha_{4}+\alpha_{5}+\alpha_{6}+\alpha_{7}+\alpha_{2},
$$$$
\alpha_{1}+2 \alpha_{3}+2 \alpha_{4}+\alpha_{5}+\alpha_{2}, \quad \alpha_{1}+\alpha_{3}+2 \alpha_{4}+\alpha_{5}+\alpha_{6}+\alpha_{2},
$$$$
\alpha_{3}+2 \alpha_{4}+2 \alpha_{5}+\alpha_{6}+\alpha_{2}, \quad \alpha_{1}+\alpha_{3}+\alpha_{4}+\alpha_{5}+\alpha_{6}+\alpha_{7}+\alpha_{2},
$$$$
\alpha_{3}+2 \alpha_{4}+\alpha_{5}+\alpha_{6}+\alpha_{7}+\alpha_{2}, \quad \alpha_{1}+2 \alpha_{3}+2 \alpha_{4}+\alpha_{5}+\alpha_{6}+\alpha_{2},
$$$$
\alpha_{1}+\alpha_{3}+2 \alpha_{4}+2 \alpha_{5}+\alpha_{6}+\alpha_{2}, \quad \alpha_{1}+\alpha_{3}+2 \alpha_{4}+\alpha_{5}+\alpha_{6}+\alpha_{7}+\alpha_{2},
$$$$
\alpha_{3}+2 \alpha_{4}+2 \alpha_{5}+\alpha_{6}+\alpha_{7}+\alpha_{2}, \quad \alpha_{1}+2 \alpha_{3}+2 \alpha_{4}+2 \alpha_{5}+\alpha_{6}+\alpha_{2},
$$$$
\alpha_{1}+2 \alpha_{3}+2 \alpha_{4}+\alpha_{5}+\alpha_{6}+\alpha_{7}+\alpha_{2}, \quad \alpha_{1}+\alpha_{3}+2 \alpha_{4}+2 \alpha_{5}+\alpha_{6}+\alpha_{7}+\alpha_{2},
$$$$
\alpha_{3}+2 \alpha_{4}+2 \alpha_{5}+2 \alpha_{6}+\alpha_{7}+\alpha_{2}, \quad \alpha_{1}+2 \alpha_{3}+3 \alpha_{4}+2 \alpha_{5}+\alpha_{6}+\alpha_{2},
$$$$
\alpha_{1}+2 \alpha_{3}+2 \alpha_{4}+2 \alpha_{5}+\alpha_{6}+\alpha_{7}+\alpha_{2}, \quad \alpha_{1}+\alpha_{3}+2 \alpha_{4}+2 \alpha_{5}+2 \alpha_{6}+\alpha_{7}+\alpha_{2},
$$$$
\alpha_{1}+2 \alpha_{3}+3 \alpha_{4}+2 \alpha_{5}+\alpha_{6}+2 \alpha_{2}, \quad \alpha_{1}+2 \alpha_{3}+3 \alpha_{4}+2 \alpha_{5}+\alpha_{6}+\alpha_{7}+\alpha_{2},
$$$$
\alpha_{1}+2 \alpha_{3}+2 \alpha_{4}+2 \alpha_{5}+2 \alpha_{6}+\alpha_{7}+\alpha_{2}, \quad \alpha_{1}+2 \alpha_{3}+3 \alpha_{4}+2 \alpha_{5}+\alpha_{6}+\alpha_{7}+2 \alpha_{2},
$$$$
\alpha_{1}+2 \alpha_{3}+3 \alpha_{4}+2 \alpha_{5}+2 \alpha_{6}+\alpha_{7}+\alpha_{2}, \quad \alpha_{1}+2 \alpha_{3}+3 \alpha_{4}+2 \alpha_{5}+2 \alpha_{6}+\alpha_{7}+2 \alpha_{2},
$$$$
\alpha_{1}+2 \alpha_{3}+3 \alpha_{4}+3 \alpha_{5}+2 \alpha_{6}+\alpha_{7}+\alpha_{2}, \quad \alpha_{1}+2 \alpha_{3}+3 \alpha_{4}+3 \alpha_{5}+2 \alpha_{6}+\alpha_{7}+2 \alpha_{2},
$$ 
We get $s_{k}$ as follows,

$$
s_{1}=34, s_{2}=15, s_{3}=29, s_{4}=55 \text {. }
$$

Then $s=55$.

The Dynkin diagram of $\mathfrak{f}_{4}$ is

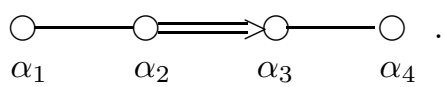

We list all positive roots

$$
\begin{aligned}
& \alpha_{1}, \quad \alpha_{2}, \quad \alpha_{3}, \quad \alpha_{4} \\
& \alpha_{1}+\alpha_{2}, \quad \alpha_{2}+\alpha_{3}, \quad \alpha_{3}+\alpha_{4} \\
& 2 \alpha_{2}+\alpha_{3}, \quad \alpha_{1}+\alpha_{2}+\alpha_{3}, \quad \alpha_{2}+\alpha_{3}+\alpha_{4} \\
& \alpha_{1}+\alpha_{2}+2 \alpha_{3}, \quad \alpha_{1}+\alpha_{2}+\alpha_{3}+\alpha_{4}, \quad \alpha_{2}+2 \alpha_{3}+\alpha_{4} \\
& \alpha_{1}+2 \alpha_{2}+2 \alpha_{3}, \quad \alpha_{2}+2 \alpha_{3}+2 \alpha_{4}, \quad \alpha_{1}+\alpha_{2}+2 \alpha_{3}+\alpha_{4} \\
& \alpha_{1}+\alpha_{2}+2 \alpha_{3}+2 \alpha_{4}, \quad \alpha_{1}+2 \alpha_{2}+2 \alpha_{3}+\alpha_{4} \\
& \alpha_{1}+2 \alpha_{2}+2 \alpha_{3}+2 \alpha_{4}, \quad \alpha_{1}+2 \alpha_{2}+3 \alpha_{3}+\alpha_{4} \\
& \alpha_{1}+2 \alpha_{2}+3 \alpha_{3}+2 \alpha_{4} \\
& \alpha_{1}+2 \alpha_{2}+4 \alpha_{3}+2 \alpha_{4} \\
& \alpha_{1}+3 \alpha_{2}+4 \alpha_{3}+2 \alpha_{4} \\
& 2 \alpha_{1}+3 \alpha_{2}+4 \alpha_{3}+2 \alpha_{4} .
\end{aligned}
$$

(18) $\mathrm{FI}, \mathfrak{g}^{\mathbb{C}}=\mathfrak{f}_{4}, l=4, r=4$.

we have

$$
\alpha_{1}^{\prime}=\lambda_{1}, \alpha_{2}^{\prime}=\lambda_{2}, \alpha_{3}^{\prime}=\lambda_{3}, \alpha_{4}^{\prime}=\lambda_{4}
$$

$\alpha^{\prime}=0$ implies $\alpha=0$.

We list $\Delta_{k}^{+}$as follows,

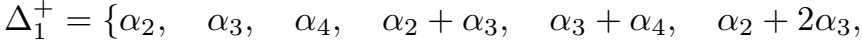

$$
\begin{aligned}
& \left.\alpha_{2}+\alpha_{3}+\alpha_{4}, \quad \alpha_{2}+2 \alpha_{3}+\alpha_{4}, \quad \alpha_{2}+2 \alpha_{3}+2 \alpha_{4}\right\} \\
& \Delta_{2}^{+}=\left\{\alpha_{1}, \quad \alpha_{3}, \quad \alpha_{4}, \quad \alpha_{3}+\alpha_{4}\right\} \\
& \Delta_{3}^{+}=\left\{\alpha_{1}, \quad \alpha_{2}, \quad \alpha_{4}, \quad \alpha_{1}+\alpha_{2}\right\} \\
& \Delta_{4}^{+}=\left\{\alpha_{1}, \quad \alpha_{2}, \quad \alpha_{3}, \quad \alpha_{1}+\alpha_{2}, \quad \alpha_{2}+\alpha_{3},\right. \\
& \left.\alpha_{2}+2 \alpha_{3}, \quad \alpha_{1}+\alpha_{2}+\alpha_{3}, \quad \alpha_{1}+\alpha_{2}+2 \alpha_{3}, \quad \alpha_{1}+2 \alpha_{2}+2 \alpha_{3}\right\} .
\end{aligned}
$$

We get $s_{k}$ as follows,

$$
s_{1}=13, s_{2}=8, s_{3}=8, s_{4}=13 \text {. }
$$

Then $s=13$.

(19) FII, $\mathfrak{g}^{\mathbb{C}}=\mathfrak{f}_{4}, l=4, r=1$.

Then $s=r=1$. 
The Dynkin diagram of $\mathfrak{g}_{2}$ is

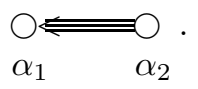

We list all positive roots

$$
\begin{array}{ll}
\alpha_{1}, \quad \alpha_{2} & \\
\alpha_{1}+\alpha_{2}, & 2 \alpha_{1}+\alpha_{2} \\
3 \alpha_{1}+\alpha_{2}, & 3 \alpha_{1}+2 \alpha_{2} .
\end{array}
$$

$(20) \mathrm{G}, \mathfrak{g}^{\mathbb{C}}=\mathfrak{g}_{2}, l=2, r=2$.

we have

$$
\alpha_{1}^{\prime}=\lambda_{1}, \alpha_{2}^{\prime}=\lambda_{2}
$$

$\alpha^{\prime}=0$ implies $\alpha=0$.

We list $\Delta_{k}^{+}$as follows,

$\Delta_{1}^{+}=\left\{\alpha_{2}\right\}$

$\Delta_{2}^{+}=\left\{\alpha_{1}\right\}$

We get $s_{k}$ as follows,

$$
s_{1}=3, s_{2}=3
$$

Then $s=3$.

Remark 4.1. For convenience, in the case of EI we give another computations by using the embedding listed in Sam $\mathrm{p} 81]$.

In $\mathbb{R}^{6}$, the positive roots of $\mathfrak{e}_{6}$ are:

$$
\varepsilon_{i}-\varepsilon_{j}, i<j ; \quad \varepsilon_{i}+\varepsilon_{j}+\varepsilon_{k}, i<j<k ; \quad \varepsilon_{1}+\varepsilon_{2}+\varepsilon_{3}+\varepsilon_{4}+\varepsilon_{5}+\varepsilon_{6} .
$$

The simple roots are

$$
\alpha_{i}=\varepsilon_{i}-\varepsilon_{i+1}, 1 \leq i \leq 5 ; \alpha_{6}=\varepsilon_{4}+\varepsilon_{5}+\varepsilon_{6} .
$$


We have

$$
\begin{aligned}
& \varepsilon_{i}-\varepsilon_{j} \quad=\alpha_{i}+\alpha_{i-1}+\cdots+\alpha_{j-1}, \quad 1 \leq i<j \leq 6 \\
& \varepsilon_{1}+\varepsilon_{2}+\varepsilon_{3}=\alpha_{1}+2 \alpha_{2}+3 \alpha_{3}+2 \alpha_{4}+\alpha_{5}+\alpha_{6} \\
& \varepsilon_{1}+\varepsilon_{2}+\varepsilon_{4}=\alpha_{1}+2 \alpha_{2}+2 \alpha_{3}+2 \alpha_{4}+\alpha_{5}+\alpha_{6} \\
& \varepsilon_{1}+\varepsilon_{2}+\varepsilon_{5}=\alpha_{1}+2 \alpha_{2}+2 \alpha_{3}+\alpha_{4}+\alpha_{5}+\alpha_{6} \\
& \varepsilon_{1}+\varepsilon_{2}+\varepsilon_{6}=\alpha_{1}+2 \alpha_{2}+2 \alpha_{3}+\alpha_{4}+\alpha_{6} \\
& \varepsilon_{1}+\varepsilon_{3}+\varepsilon_{4}=\alpha_{1}+\alpha_{2}+2 \alpha_{3}+2 \alpha_{4}+\alpha_{5}+\alpha_{6} \\
& \varepsilon_{1}+\varepsilon_{3}+\varepsilon_{5}=\alpha_{1}+\alpha_{2}+2 \alpha_{3}+\alpha_{4}+\alpha_{5}+\alpha_{6} \\
& \varepsilon_{1}+\varepsilon_{3}+\varepsilon_{6}=\alpha_{1}+\alpha_{2}+2 \alpha_{3}+\alpha_{4}+\alpha_{6} \\
& \varepsilon_{1}+\varepsilon_{4}+\varepsilon_{5}=\alpha_{1}+\alpha_{2}+\alpha_{3}+\alpha_{4}+\alpha_{5}+\alpha_{6} \\
& \varepsilon_{1}+\varepsilon_{4}+\varepsilon_{6}=\alpha_{1}+\alpha_{2}+\alpha_{3}+\alpha_{4}+\alpha_{6} \\
& \varepsilon_{1}+\varepsilon_{5}+\varepsilon_{6}=\alpha_{1}+\alpha_{2}+\alpha_{3}+\alpha_{6} \\
& \varepsilon_{2}+\varepsilon_{3}+\varepsilon_{4}=\alpha_{2}+2 \alpha_{3}+2 \alpha_{4}+\alpha_{5}+\alpha_{6} \\
& \varepsilon_{2}+\varepsilon_{3}+\varepsilon_{5}=\alpha_{2}+2 \alpha_{3}+\alpha_{4}+\alpha_{5}+\alpha_{6} \\
& \varepsilon_{2}+\varepsilon_{3}+\varepsilon_{6}=\alpha_{2}+2 \alpha_{3}+2 \alpha_{4}+\alpha_{6} \\
& \varepsilon_{2}+\varepsilon_{4}+\varepsilon_{5}=\alpha_{2}+\alpha_{3}+\alpha_{4}+\alpha_{5}+\alpha_{6} \\
& \varepsilon_{2}+\varepsilon_{4}+\varepsilon_{6}=\alpha_{2}+\alpha_{3}+\alpha_{4}+\alpha_{6} \\
& \varepsilon_{2}+\varepsilon_{5}+\varepsilon_{6}=\alpha_{2}+\alpha_{3}+\alpha_{6} \\
& \varepsilon_{3}+\varepsilon_{4}+\varepsilon_{5}=\alpha_{3}+\alpha_{4}+\alpha_{5}+\alpha_{6} \\
& \varepsilon_{3}+\varepsilon_{4}+\varepsilon_{6}=\alpha_{3}+\alpha_{4}+\alpha_{6} \\
& \varepsilon_{3}+\varepsilon_{5}+\varepsilon_{6}=\alpha_{3}+\alpha_{6} \\
& \varepsilon_{4}+\varepsilon_{5}+\varepsilon_{6}=\alpha_{6} \\
& \varepsilon_{1}+\varepsilon_{2}+\varepsilon_{3}+\varepsilon_{4}+\varepsilon_{5}+\varepsilon_{6}=\alpha_{1}+2 \alpha_{2}+3 \alpha_{3}+2 \alpha_{4}+\alpha_{5}+2 \alpha_{6} \text {. }
\end{aligned}
$$

To get $\Delta_{k}^{+}$, we have two cases.

For $\alpha=\varepsilon_{i}-\varepsilon_{j}, i<j, m_{k}^{\prime}(\alpha)=0$, then $i<j \leq k$ or $j>i>k$, the number of roots is

$$
\left(\begin{array}{l}
k \\
2
\end{array}\right)+\left(\begin{array}{c}
6-k \\
2
\end{array}\right) .
$$

For $\alpha=\varepsilon_{i}+\varepsilon_{j}+\varepsilon_{p}, i<j<p, m_{k}^{\prime}(\alpha)=0$, then we have

$$
\begin{aligned}
k= & 1,2,3, \quad k<i<j<p, \text { the number of positive roots is }\left(\begin{array}{c}
6-k \\
3
\end{array}\right) . \\
k= & 4, \alpha_{1}+\alpha_{5}+\alpha_{6}, \alpha_{2}+\alpha_{5}+\alpha_{6}, \alpha_{3}+\alpha_{5}+\alpha_{6}, \alpha_{4}+\alpha_{5}+\alpha_{6} . \\
k= & 5, \alpha_{1}+\alpha_{2}+\alpha_{6}, \alpha_{1}+\alpha_{3}+\alpha_{6}, \alpha_{1}+\alpha_{4}+\alpha_{6}, \alpha_{1}+\alpha_{5}+\alpha_{6}, \alpha_{2}+\alpha_{3}+\alpha_{6}, \\
& \alpha_{2}+\alpha_{4}+\alpha_{6}, \alpha_{2}+\alpha_{5}+\alpha_{6}, \alpha_{3}+\alpha_{4}+\alpha_{6}, \alpha_{3}+\alpha_{5}+\alpha_{6}, \alpha_{4}+\alpha_{5}+\alpha_{6} .
\end{aligned}
$$

$k=6$, empty.

Then we get the values of $s_{k}$ are $26,17,13,17,26,21$ respectively. 
Now we get all s-values for irreducible Riemannian symmetric spaces of compact type. In summary, we have the table 1.1 in the introduction of the present paper.

\section{REFERENCES}

[Frv] H. Freudenthal and H. de Vris, Linear Lie groups, Pure Appl. Math. 35, Academic Press, New York, 1969.

[Fr1] T.Frankel, Manifolds with positive curvature, Pacific Jour. Math., 11(1961)165-174.

[Fr2] T.Frankel, On the fundmental group of minimal submanifolds, Ann. of Math., 83(1966)68-73.

[Kx1] K.Kenmotsu and C.Y.Xia, Intersections of minimal submanifolds in Riemannian manifold with partially positive curvature, Kodai Math. J., 18(1995)242-249.

[Kx2] K.Kenmotsu and C.Y.Xia, Hadamard-Frankel type theorems for manifolds with partially positive curvature, Pacific Journal Math., 176(1996)129-139.

[Kna] A.W.Knapp, Lie groups, Beyond an introduction, Second edition, Progress in mathematics, volume 140, 2002.

[Wal] N.R.Wallach, On maximal subsystems of root systems, Canad. J. Math., 20(1968)555-574.

[Hel] S. Helgason, Differential geometry, Lie groups, and Symmetric spaces, Graduate Studies in Mathematics, volume 34, 2001.

[Lee1] Nany Lee, on the lower bound of the mean curvature of constant mean curvature hypersurface in non-compact symmetric spaces and manifolds with a pole, Tohoku Math. Jour., (2)47(1995)499-508.

[Lee2] Nany Lee, Determination of the partial positivity of the curvature in symmetric spaces, Annali di Matimatica pura ed applicata, 171(1996)107-129.

[Sam] H. Samelson, Notes on Lie algebras, Springer-Verlag Universitext, 1989.

[Shz] Z. Shen, On complete manifolds of nonnegative kth-Ricci curvature, Trans. Amer. Math. Soc., 338(1993)289-310.

[Wu] H.Wu, Manifolds of partially positive curvature, Indiana Univ. Math. J., 36(1987)525-548. 